EURANDOM PREPRINT SERIES

2011-039 November, 2011

Erlang arrivals joining the shorter queue

I.J.B.F. Adan, S. Kapodistria, J.S.H. van Leeuwaarden ISSN 1389-2355 


\title{
Erlang arrivals joining the shorter queue
}

\author{
Ivo J.B.F. Adan* Stella Kapodistria* Johan S.H. van Leeuwaarden*
}

November 2, 2011

\begin{abstract}
We consider a system in which customers join upon arrival the shortest of two single-server queues. The interarrival times between customers are Erlang distributed and the service times of both servers are exponentially distributed. Under these assumptions, this system gives rise to a Markov chain on a multi-layered quarter plane. For this Markov chain we derive the equilibrium distribution using the compensation approach. The obtained expression for the equilibrium distribution matches and refines heavy-traffic approximations and tail asymptotics obtained earlier in the literature.
\end{abstract}

Keywords: random walks in the quarter plane, compensation approach, join the shorter queue, tail asymptotics

AMS 2000 Subject Classification: 60K25, 90B22

\section{Introduction}

Consider a system of two unbounded single server queues, in which a customer on arrival joins the shorter queue. The service times of customers are independent and exponentially distributed with mean one, irrespective of the queue they have joined. The arrivals form a renewal process with Erlang interarrival times with mean $k / \lambda$, so that each interarrival time consists of $k$ independent exponential phases with mean $1 / \lambda$. Under these Markovian assumptions, the evolution of the system can be described as a three-dimensional Markov chain, whose state space consists of a $k$-layered quarter plane. When $\rho=\lambda / 2 k<1$ this Markov chain is ergodic and the equilibrium distribution exists. In this paper we derive an explicit expression for the equilibrium distribution in terms of an infinite series of geometric terms. This explicit expression immediately renders heavy-traffic approximations valid when $\rho$ is close to unity, including the asymptotic result that connects the tail exponent of the shortest queue length to the tail exponent of the corresponding queueing system with Erlang arrivals, one common queue and two servers.

The shorter queue problem with Poisson arrivals $(k=1)$ was originally proposed by Haight [14]. Kingman [15] came up with an ingenious approach for finding the equilibrium distribution. Kingman first transformed the state space such that the queue length process was described by a random walk in the quarter plane, next described the bivariate generating function of the equilibrium distribution

\footnotetext{
${ }^{1}$ Department of Mathematics and Computer Science, Eindhoven University of Technology, P.O. Box 513, 5600 MB Eindhoven, The Netherlands

E-mails: i.j.b.f.adan@tue.nl, s.kapodistria@tue.nl, j.s.h.v.leeuwaarden@tue.nl
} 
in terms of a functional equation, and finally solved the functional equation using complex analysis. Kingman's work was the first of many papers exploiting the connection between two-dimensional random walks in the quarter plane and functional equations. For the general class of random walks in the quarter plane Malyshev pioneered this approach in the 1970's, and the theory has advanced since, via applications like lattice path counting and two-server queueing models. The crucial idea was to reduce the functional equations to standard boundary value problems, and extensive treatments of this technique can be found in Cohen and Boxma [8] and Fayolle, Iasnogorodski and Malyshev [9]. It typically concerns sophisticated complex analysis, Riemann surfaces and conformal mappings.

Kingman's solution to the functional equation for the shortest queue problem, however, was simple and elegant, and only required an iterative mapping that was shown to lead to a convergent solution. In fact, it was shown later that Kingman's solution could be obtained without using the functional equation, and by solving the equilibrium equations directly. This direct method was called the compensation approach and developed in a series of papers $[1,3,5]$. It exploits the fact that the (linear) equilibrium equations in the interior of the quarter plane are satisfied by linear combinations of product-forms, the parameters of which satisfy a kernel equation, and that need to be chosen such that the equilibrium equations on the boundaries are satisfied as well. As it turns out, this can be done by alternatingly compensating for the errors on the two boundaries, which eventually leads to an infinite series of product-forms that matches the solution of Kingman. In $[1,3,5]$, the compensation approach has been shown to work for two-dimensional random walks on the lattice of the first quadrant that obey the following conditions:

- Step size: Only transitions to neighboring states.

- Forbidden steps: No transitions from interior states to the North, North-East, and East.

- Homogeneity: The same transitions occur according to the same rates for all interior points, and similarly for all points on the horizontal boundary, and for all points on the vertical boundary.

Although the theory has been developed for two-dimensional random walks with only one layer, this paper demonstrates that it can also be applied to random walks with multiple layers. For this, we have to extend the compensation approach to a three-dimensional setting.

Random walks in the quarter plane play the role of canonical example for obtaining tail asymptotics for equilibrium distributions. Indeed, random walks in the quarter plane present serious challenges to the traditional approaches for obtaining tail asymptotics, including large-deviations theory, matrixanalytic methods, and complex-function methods. A comprehensive overview of the state of the art in this field is given in [21]. Our contribution to this area is that, for the special case of the shorter queue model with Erlang arrivals, we obtain an exact expression for the equilibrium distribution, which by its nature, also reveals the tail asymptotics, including leading-order behavior, correction terms and explicit error estimates. Our exact results yield asymptotic results that match and refine those obtained in [24].

The paper is organized as follows. In Section 2 we describe the model in full detail. In Section 3 we present a first result on the decay rate of the shortest queue length. In Section 4 we develop the three-dimensional compensation approach. In Section 5 we present the explicit expression for the equilibrium distribution. Finally, in Section 6 we present some conclusions. 


\section{Model description}

Consider a system consisting of two identical servers, in which the service times of customers are independent and exponentially distributed with mean one, irrespective of the queue they have joined. The interarrival times between consecutive jobs follow an Erlang distribution with mean $k / \lambda$, consisting of $k$ exponential phases, each with mean $1 / \lambda$. Upon arrival a customer joins the shortest queue, and in case of a tie, the customer joins either queue with probability $1 / 2$. This system is stable if and only if $\rho=\lambda / 2 k<1$. The stability condition can be easily proven as follows. We know that the number of jobs in this shortest queue system is stochastically larger than in the $E_{k} / M / 2$ queue with FCFS discipline (see [12] and the references therein). On the other hand it is stochastically smaller than in the system, where each server has his own waiting line and customers are assigned to either line with probability $1 / 2$ (see [17]). Since both bounding models have the same stability condition $\rho<1$, we can conclude that this condition is necessary and sufficient to ensure stability for the model at hand.

An Erlang arrival process corresponds with the assumption that each customers first goes through $k$ independent exponential phases before arriving to the system. Let $R(t)$ denote the phase in which the customer to arrive next is in at time $t$. Further, let $Q_{i}(t)$ denote the stochastic process describing the number of jobs in queue $i$ at time $t$. For convenience introduce $X_{1}(t)=\min \left\{Q_{1}(t), Q_{2}(t)\right\}$ and $X_{2}(t)=$ $\left|Q_{1}(t)-Q_{2}(t)\right|$. Then the dynamics of the queueing system can be described by a continuous-time Markov chain $\left\{\left(R(t), X_{1}(t), X_{2}(t)\right), t \geq 0\right\}$, with state space $\{(h, m, n), h=0,1, \ldots, k-1, m, n=$ $0,1, \ldots\}$, where $h$ is the number of completed phases of arrival, $m$ the length of the shortest queue and $n$ the difference between the longest and the shortest queue. Under the stability condition $\rho=\lambda / 2 k<1$, we shall determine the equilibrium distribution

$$
p(h, m, n)=\lim _{t \rightarrow \infty} \mathbb{P}\left(\left(R(t), X_{1}(t), X_{2}(t)\right)=(h, m, n)\right), h=0,1, \ldots, k-1, m, n=0,1, \ldots
$$

of this three-dimensional Markov chain.

The transition rates are given by

$$
\begin{aligned}
& (h, m, n) \stackrel{2 k \rho}{\longrightarrow}(h+1, m, n), h=0, \ldots, k-2, m \geq 0, n \geq 0, \\
(k-1, m, n) & \stackrel{2 k \rho}{\longrightarrow}(0, m+1, n-1), m \geq 0, n \geq 1, \\
(k-1, m, 0) & \stackrel{2 k \rho}{\longrightarrow}(0, m, 1), m \geq 0,
\end{aligned}
$$

corresponding to arrivals, and

$$
\begin{aligned}
& (h, m, n) \stackrel{1}{\longrightarrow}(h, m-1, n+1), h=0, \ldots, k-1, m \geq 1, n \geq 1, \\
& (h, m, n) \stackrel{1}{\longrightarrow}(h, m, n-1), h=0, \ldots, k-1, m \geq 0, n \geq 1, \\
& (h, m, 0) \stackrel{2}{\longrightarrow}(h, m-1,1), h=0, \ldots, k-1, m \geq 1,
\end{aligned}
$$




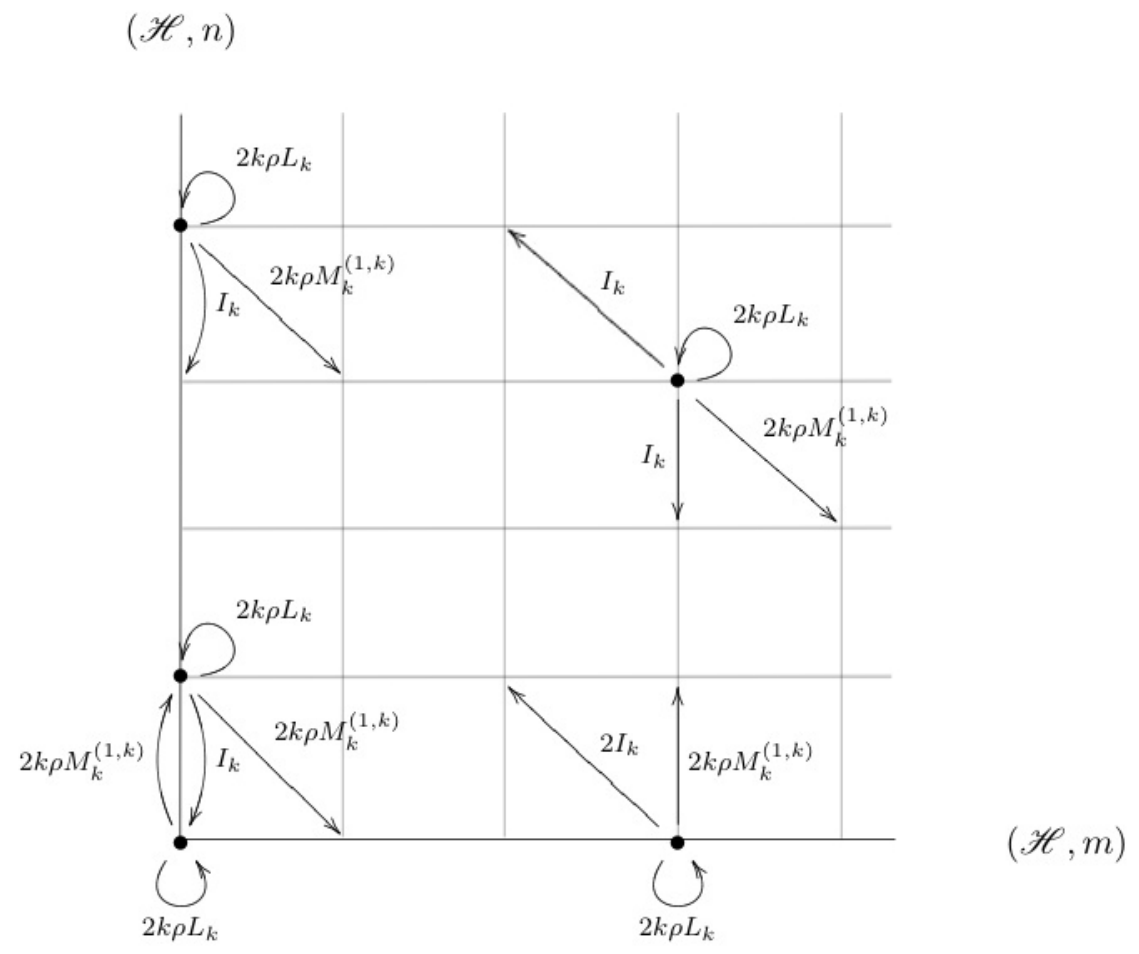

Figure 1: $k$-layered transition rate diagram with $\mathscr{H}=\{0,1, \ldots, k-1\}$.

corresponding to service completions. The equilibrium equations then read

$$
\begin{aligned}
2 k \rho p(h, 0,0) & =p(h, 0,1)+2 k \rho\left(1-\delta_{h, 0}\right) p(h-1,0,0), \\
(2 k \rho+1) p(h, 0,1) & =p(h, 0,2)+2 p(h, 1,0)+2 k \rho \delta_{h, 0} p(k-1,0,0) \\
& +2 k \rho\left(1-\delta_{h, 0}\right) p(h-1,0,1) \\
(2 k \rho+1) p(h, 0, n) & =p(h, 1, n-1)+p(h, 0, n+1) \\
& +2 k \rho\left(1-\delta_{h, 0}\right) p(h-1,0, n), n \geq 2, \\
2(k \rho+1) p(h, m, 0) & =p(h, m, 1)+2 k \rho \delta_{h, 0} p(k-1, m-1,1) \\
& +2 k \rho\left(1-\delta_{h, 0}\right) p(h-1, m, 0), m \geq 1, \\
2(k \rho+1) p(h, m, 1) & =p(h, m, 2)+2 p(h, m+1,0)+2 k \rho \delta_{h, 0} p(k-1, m-1,2) \\
& +2 k \rho \delta_{h, 0} p(k-1, m, 0)+2 k \rho\left(1-\delta_{h, 0}\right) p(h-1, m, 1), m \geq 1, \\
2(k \rho+1) p(h, m, n) & =p(h, m, n+1)+p(h, m+1, n-1)+2 k \rho \delta_{h, 0} p(k-1, m-1, n+1) \\
& +2 k \rho\left(1-\delta_{h, 0}\right) p(h-1, m, n), m \geq 1, n \geq 2,
\end{aligned}
$$

where $h=0, \ldots, k-1$ and $\delta_{i, j}$ denotes the Kronecker delta taking value 1 when $i=j$ and 0 otherwise. Let $\boldsymbol{p}(m, n)=(p(0, m, n), p(1, m, n), \ldots, p(k-1, m, n))^{T}$, with $\boldsymbol{x}^{T}$ the transpose of a column vector $\boldsymbol{x}$. We introduce the following matrix notation to describe the equilibrium equations:

$$
\begin{aligned}
& \boldsymbol{A}_{0,0} \boldsymbol{p}(0,0)+\boldsymbol{A}_{0,-1} \boldsymbol{p}(0,1)=\mathbf{0} \\
& \boldsymbol{B}_{0,0} \boldsymbol{p}(0,1)+\boldsymbol{A}_{0,-1} \boldsymbol{p}(0,2)+2 \boldsymbol{A}_{-1,1} \boldsymbol{p}(1,0)+\boldsymbol{A}_{0,1} \boldsymbol{p}(0,0)=\mathbf{0}
\end{aligned}
$$




$$
\begin{aligned}
& \boldsymbol{B}_{0,0} \boldsymbol{p}(0, n)+\boldsymbol{A}_{0,-1} \boldsymbol{p}(0, n+1)+\boldsymbol{A}_{-1,0} \boldsymbol{p}(1, n-1)=\mathbf{0}, n \geq 2, \\
& \boldsymbol{C}_{0,0} \boldsymbol{p}(m, 0)+\boldsymbol{A}_{0,-1} \boldsymbol{p}(m, 1)+ \boldsymbol{A}_{1,-1} \boldsymbol{p}(m-1,1)=\mathbf{0}, m \geq 1, \\
& \boldsymbol{C}_{0,0} \boldsymbol{p}(m, 1)+\boldsymbol{A}_{0,-1} \boldsymbol{p}(m, 2)+ 2 \boldsymbol{A}_{-1,1} \boldsymbol{p}(m+1,0)+\boldsymbol{A}_{1,-1} \boldsymbol{p}(m-1,2) \\
&+\boldsymbol{A}_{0,1} \boldsymbol{p}(m, 0)=\mathbf{0}, m \geq 1, \\
& \boldsymbol{C}_{0,0} \boldsymbol{p}(m, n)+\boldsymbol{A}_{0,-1} \boldsymbol{p}(m, n+1)+\boldsymbol{A}_{-1,1} \boldsymbol{p}(m+1, n-1) \\
&+\boldsymbol{A}_{1,-1} \boldsymbol{p}(m-1, n+1)=\mathbf{0}, m \geq 1, n \geq 2,
\end{aligned}
$$

where

$$
\begin{aligned}
\boldsymbol{A}_{0,-1} & =\boldsymbol{A}_{-1,1}=\boldsymbol{A}_{-1,0}=\boldsymbol{I}_{k}, \\
\boldsymbol{A}_{0,1} & =\boldsymbol{A}_{1,-1}=2 k \rho \boldsymbol{M}_{k}^{(1, k)}, \\
\boldsymbol{A}_{0,0} & =-2 k \rho \boldsymbol{I}_{k}+2 k \rho \boldsymbol{L}_{k}, \\
\boldsymbol{B}_{0,0} & =-(2 k \rho+1) \boldsymbol{I}_{k}+2 k \rho \boldsymbol{L}_{k}, \\
\boldsymbol{C}_{0,0} & =-2(k \rho+1) \boldsymbol{I}_{k}+2 k \rho \boldsymbol{L}_{k},
\end{aligned}
$$

with $\boldsymbol{I}_{k}$ the $k \times k$ identity matrix, $\boldsymbol{M}_{k}^{(i, j)}$ a $k \times k$ binary matrix with element $(i, j)$ equal to one and zeros elsewhere, and $\boldsymbol{L}_{k}$ a lower shift matrix with elements $(i, i-1), i=2, \ldots, k$, equal to one and zeros elsewhere.

\section{Decay rate}

Sakuma et al. [24] studied the join the shorter queue model with phase-type arrivals (of which Erlang arrivals are a special case). They combined matrix-analytic techniques with general results for decay rates of quasi-birth-death processes to establish the following result. Although the result in [24] is more general, here we present the result for the special case of Erlang arrivals. Let $\sigma$ denote the unique positive real root of $\sigma=\tilde{A}(2(1-\sigma))$ inside the open unit circle, with $\tilde{A}(s)$ the Laplace-Stieltjes transform of the interarrival time distribution.

Proposition 3.1 (Sakuma et al. [24]) For $h=0,1, \ldots, k-1$,

$$
\lim _{m \rightarrow \infty} \sigma^{-2 m} p(h, m, n)=K_{h, 1} \beta_{1}^{n}+K_{h, 2} \beta_{2}^{n}+\cdots+K_{h, k} \beta_{k}^{n}
$$

with $\beta_{1}, \ldots, \beta_{k}$ and $K_{h, 1}, \ldots, K_{h, k}$ constants that do not depend on $m$.

Proposition 3.1 can be understood by comparing the join the shorter queue model with the corresponding $G / M / 2$ model with one queue, two servers, phase-type arrivals with mean interarrival time $1 / 2 \rho$ and mean service time one. Let $Q_{1}$ and $Q_{2}$ denote the steady-state queue length in the original join the shorter queue model, and let $Q$ denote the steady-state queue length in the $G / M / 2$ model with one queue. It is then expected that $\mathbb{P}\left(Q_{1}+Q_{2}=m\right)$ and $\mathbb{P}(Q=m)$ have the same decay rate, since both systems will work at full capacity whenever the total number of customers grows large. Moreover, since the join the shorter queue discipline constantly aims at balancing the lengths of the two queues over time, it is expected that, for large values of $m$,

$$
\mathbb{P}\left(\min \left(Q_{1}, Q_{2}\right)=m\right) \approx \mathbb{P}\left(Q_{1}+Q_{2}=2 m\right) \approx \mathbb{P}(Q=2 m) .
$$


For the standard $G / M / 2$ model with one queue, it is well known that

$$
\mathbb{P}(Q=m) \approx(1-\sigma) \sigma^{m},
$$

where $\sigma$ is the unique root in $(0,1)$ of $\sigma=\tilde{A}(2(1-\sigma))$, with $\tilde{A}(s)$ the Laplace-Stieltjes transform of the interarrival distribution. Notice that in case of Erlang arrivals this gives

$$
\sigma=\left[\frac{k \rho}{k \rho+1-\sigma}\right]^{k}
$$

Combining (3.2) and (3.3) leads to the following conjectured behavior of the tail probability of the minimum queue length;

$$
\mathbb{P}\left(\min \left(Q_{1}, Q_{2}\right)=m\right) \approx c \sigma^{2 m}, m \rightarrow \infty,
$$

for some positive constant $c$. Hence, the decay rate of the tail probabilities for $\min \left(Q_{1}, Q_{2}\right)$ is conjectured to equal the square of the decay rate of the tail probabilities of $Q$. Proposition 3.1 thus proves this conjecture, for the case when the difference of the queue sizes and the arrival phase are fixed. In [24] it was left as an open problem to determine the decay rate of the marginal distribution of $\min \left(Q_{1}, Q_{2}\right)$. The latter does not follow immediately from Proposition 3.1, because the summation of the difference in queue sizes, which can be unbounded, requires a formal justification. In this paper, we derive an exact expression for $p(h, m, n)$, which, among other things renders the following result.

\section{Proposition 3.2}

$$
\lim _{m \rightarrow \infty} \sigma^{-2 m} \mathbb{P}\left(\min \left(Q_{1}, Q_{2}\right)=m\right)=\sum_{j=1}^{k} \frac{\kappa_{j}}{1-\beta_{j}}
$$

with $\kappa_{j}=\sum_{h=0}^{k-1} K_{h, j}$.

\section{The compensation procedure}

In this section we shall present a series of preliminary results that together will lead up to the full solution of the equilibrium equations reported in Section 5. We start by exploiting the intuition developed in Section 3 about the tail asymptotics in (3.5). In order to establish that the equilibrium distribution $p(h, m, n)$ is indeed of a form that would yield the asymptotics of the type (3.5) we first consider a modified model that is closely related to the original model and that is expected to give the same asymptotic behavior.

The modified model is constructed as follows. We start from the state space of one model illustrated in Fig. 1 and bend the vertical axis as shown in Fig. 2 to create a modified model that has the same equilibrium equations in the interior and on the horizontal boundary. This model can be recognized as the Erlang arrivals joining the shorter queue in a two-server production system with the additional feature that when one of the queues is left empty the server works in advance and puts the produced items on stock. Then, when a customer arrives he takes immediately a product from the stock and leaves the system, and when both queues are empty again, the stock is cleared.

The characteristic feature of the modified model is that its equilibrium equations for $m+n=0$ are exactly the same as the ones in the interior; in this sense the modified model has no "vertical" boundary equations. 


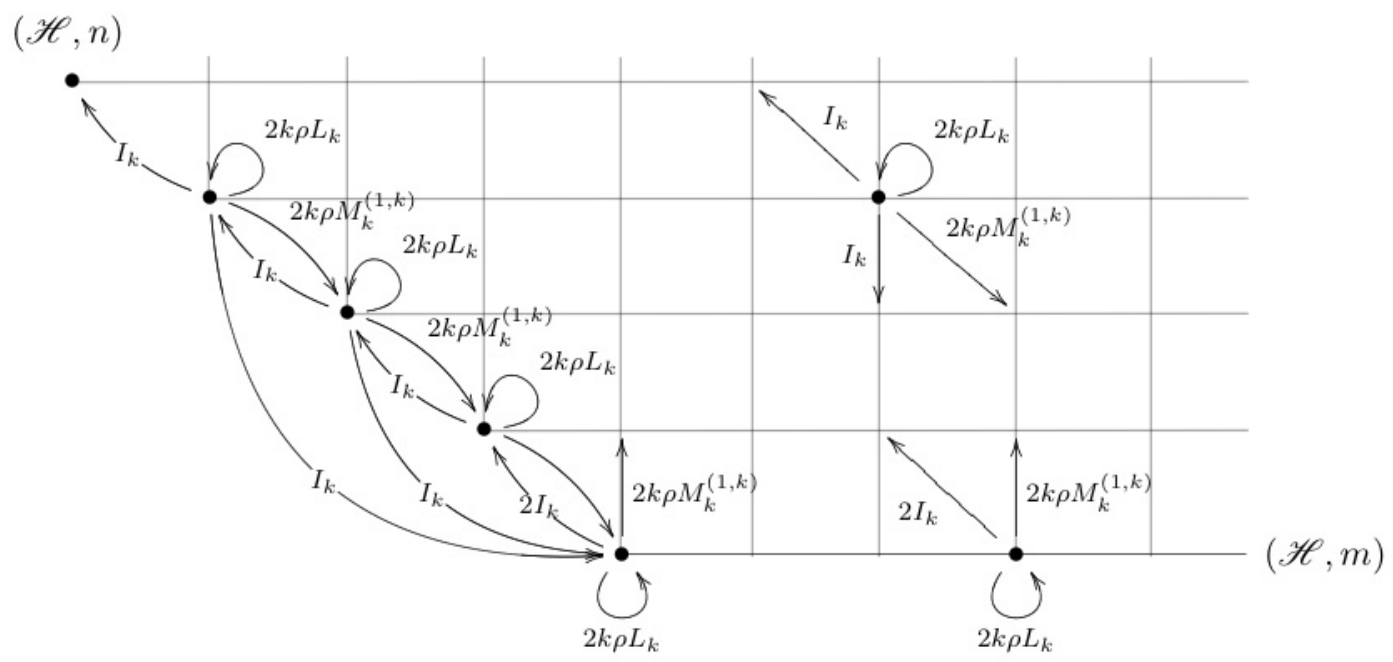

Figure 2: $k$-layered transition rate diagram for the modified model with $\mathscr{H}=\{0,1, \ldots, k-1\}$.

We now present two lemmas. The first lemma gives a product-form solution for the modified model. The second lemma gives a similar product-form solution for the original model via exploiting the relation with the modified model. Let $\hat{p}(h, m, n)$ denote the equilibrium distribution of the modified model.

Lemma 4.1 For $\rho<1$, the equilibrium distribution of the modified model exists and it is of the form

$$
\hat{p}(h, m, n)=\alpha^{m} \hat{q}_{h}(n), h=0,1, \ldots, k-1, m \geq-n, n \geq 0,
$$

with $\alpha \in(0,1)$ unique and $\left\{\hat{q}_{h}(n)\right\}_{n \in \mathbb{N}_{0}}$ such that $\sum_{n=0}^{\infty} \alpha^{-n} \hat{q}_{h}(n)<\infty$.

Proof. First notice that the modified model is stable whenever the original model is stable and that $\hat{p}(h, m, n)$ satisfies (2.10)-(2.12) for all $m+n \geq 0$, except for states $(h, 0,0)$. Observe that the modified model, restricted to an area of the form $\left\{(m, n): m \geq n_{0}-n, n \geq 0, n_{0}=1,2, \ldots\right\}$ embarked by a line parallel to the diagonal axis, yields the exact same process. Hence, we can conclude that for $\hat{\boldsymbol{p}}(m, n)=(\hat{p}(0, m, n), \ldots, \hat{p}(k-1, m, n))^{T}$,

$$
\hat{\boldsymbol{p}}(m+1, n)=\alpha \hat{\boldsymbol{p}}(m, n), m \geq-n, n \geq 0,
$$

and therefore

$$
\hat{p}(h, m, n)=\alpha^{m} \hat{q}_{h}(n), h=0,1, \ldots, k-1, m \geq-n, n \geq 0 .
$$

Finally, we observe that

$$
\sum_{n=0}^{\infty} \alpha^{-n} \hat{q}_{h}(n)=\sum_{n=0}^{\infty} \hat{p}(h,-n, n)<1
$$

which concludes the proof.

In Lemma 4.1 we have shown that the equilibrium distribution of the modified model has a productform expression which is unique up to a positive multiplicative constant. Furthermore, one can verify that the unique $\alpha$ of formula (4.1) is equal to $\sigma^{2}$, where $\sigma$ is the unique root of (3.4) in the interval 
$(0,1)$ (see Appendix A). Returning to the original model, we will prove completely independently from Lemma 4.1 that the solution of the equilibrium equations (2.10)-(2.12) is identical to the expression for the modified model as given in (4.1), with $\alpha=\sigma^{2}$.

Lemma 4.2 For $\rho<1$, there exists a product-form

$$
\boldsymbol{p}(m, n)=\sigma^{2 m} \boldsymbol{q}(n), m, n \geq 0,
$$

with $\sigma$ the unique root of (3.4) inside the open unit circle and $\boldsymbol{q}(n)=\left(q_{0}(n), \ldots, q_{k-1}(n)\right)^{T}$ non-zero such that $\sum_{n=0}^{\infty} \sigma^{-2 n} q_{h}(n)<\infty, h=0, \ldots, k-1$, which satisfies the equilibrium equations (2.10)(2.12).

Proof. Substituting $\boldsymbol{p}(m, n)=\sigma^{2 m} \boldsymbol{q}(n)$ into the equilibrium equations (2.10)-(2.12) reveals that $\boldsymbol{q}(n)$ satisfies a system of linear difference equations. Let $\boldsymbol{Q}(y)=\left(Q_{0}(y), \ldots, Q_{k-1}(y)\right)^{T}$, with $Q_{h}(y)=$ $\sum_{n=0}^{\infty} y^{n} q_{h}(n),|y|<1 / \sigma^{2}$. It then follows after straightforward calculations that

$$
\boldsymbol{H}_{1}(y) \boldsymbol{Q}(y)=\boldsymbol{H}_{2}(y) \boldsymbol{Q}(0)
$$

with

$$
\begin{aligned}
& \boldsymbol{H}_{1}(y)=\left(-2(k \rho+1) \sigma^{2} y+\sigma^{2}+\sigma^{4} y^{2}\right) \boldsymbol{I}_{k}+2 k \rho \sigma^{2} y \boldsymbol{L}_{k}+2 k \rho \boldsymbol{M}_{k}^{(1, k)}, \\
& \boldsymbol{H}_{2}(y)=\sigma^{2}\left(1-\sigma^{2} y^{2}\right) \boldsymbol{I}_{k}+2 k \rho\left(1-\sigma^{2} y^{2}\right) \boldsymbol{M}_{k}^{(1, k)} .
\end{aligned}
$$

We will show that $\boldsymbol{Q}(y)$ is non-zero and analytic within the circle of radius $1 / \sigma^{2}$.

In order to analyze the linear system (4.5) we should investigate for which values of $y$ the matrix $\boldsymbol{H}_{1}(y)$ is not invertible. Setting $\operatorname{det}\left(\boldsymbol{H}_{1}(y)\right)=0$, for $\sigma \neq 0$, is tantamount to

$$
\left(2(k \rho+1) \sigma^{2} y-\sigma^{2}-\left(\sigma^{2} y\right)^{2}\right)^{k}-(2 k \rho)^{k}\left(\sigma^{2} y\right)^{k-1}=0
$$

First, we will show that equation (4.6) has $k$ roots inside the open circle of radius $1 / \sigma^{2}$. To this end, we define $u=\sigma^{2} y$ and observe that (4.6) can be written as

$$
h_{1}^{k}(u)-(2 k \rho)^{k} u^{k-1}=0
$$

with $h_{1}(u)=2(k \rho+1) u-\sigma^{2}-u^{2}$. Then $h_{1}(u)$ has two roots:

$$
u^{ \pm}=k \rho+1 \pm \sqrt{(k \rho+1)^{2}-\sigma^{2}} .
$$

Observe that, for $0<\rho<1$

$$
\left|u^{+}\right|=(k \rho+1)\left|1+\sqrt{1-\frac{\sigma^{2}}{(k \rho+1)^{2}}}\right|>k \rho+1>1,
$$

and

$$
\left|u^{-}\right|=\frac{\sigma^{2}}{\left|u^{+}\right|}<|\alpha|<1
$$


Furthermore, for $|u|=1$ one can easily see that

$$
\begin{aligned}
\left|h_{1}^{k}(u)\right| & =\left|2(k \rho+1) u-\sigma^{2}-u^{2}\right|^{k} \geq\left.\left.|2(k \rho+1)| u\left|-\sigma^{2}-\right| u\right|^{2}\right|^{k} \\
& =\left(2 k \rho+1-\sigma^{2}\right)^{k}>(2 k \rho)^{k} .
\end{aligned}
$$

Hence, by Rouché's theorem, $h_{1}^{k}\left(\sigma^{2} y\right)-(2 k \rho)^{k}\left(\sigma^{2} y\right)^{k-1}$ has exactly $k$ roots, denoted by $y_{1}, y_{2}, \ldots, y_{k}$, inside the circle of radius $1 / \sigma^{2}$.

Next, we will show that these roots are distinct. The assumption that $y_{i}=y_{j}$ for some $i, j=1, \ldots, k$, with $i \neq j$, or equivalently $u_{i}=\sigma^{2} y_{i}=\sigma^{2} y_{j}=u_{j}$ for $i \neq j$, contradicts $\sigma^{2} \in(0,1)$. To prove this contradiction observe that, for a root of $h_{1}^{k}\left(\sigma^{2} y\right)-(2 k \rho)^{k}\left(\sigma^{2} y\right)^{k-1}$ in (4.6) to be of (at least) multiplicity two, it must be that $h_{1}^{k}(u)-(2 k \rho)^{k} u^{k-1}=k h_{1}^{k-1}(u) h_{1}^{\prime}(u)-(k-1)(2 k \rho)^{k} u^{k-2}=0$, which gives

$$
\sigma^{2}=u \frac{(k+1) u-2(k \rho+1)}{k-1} .
$$

Substituting (4.8) into (4.6) yields

$$
u(k \rho+1-y)^{k}-(k-1)^{k} \rho^{k}=0 .
$$

Equation (4.9) has according to Rouché's theorem a unique solution inside $|u|=\rho$, since

$$
\begin{aligned}
\left|u-\left(\frac{(k-1) \rho}{k \rho+1-u}\right)^{k}-u\right| & =\left|\left(\frac{(k-1) \rho}{k \rho+1-u}\right)^{k}\right| \leq\left(\frac{(k-1) \rho}{k \rho+1-|u|}\right)^{k} \\
& <\left(\frac{(k-1) \rho}{(k-1) \rho+1}\right)^{k}<\rho, \text { for all } k \geq 2
\end{aligned}
$$

Furthermore, applying the intermediate value theorem in the interval $[0, \rho]$ shows that the solution is real. Then, for $u \leq \rho$ the $\sigma^{2}$ we obtain from (4.8) is a non-positive number, which contradicts the assumption that $\sigma^{2} \in(0,1)$.

We have shown that (4.6) has $k$ roots inside the open circle of radius $1 / \sigma^{2}$, denoted by $y_{1}, \ldots, y_{k}$. Furthermore, one can easily verify that one of these roots is equal to $1 / \sigma$, say $y_{k}=1 / \sigma$, and observe that this is the unique root (with multiplicity $k$ ) of the determinant of matrix $\boldsymbol{H}_{2}(y)$. The rest of the roots $y_{1}, \ldots, y_{k-1}$ will be used to determine the vector $\boldsymbol{Q}(0)$ up to a non-zero constant. More concretely, for the roots $y_{1}, \ldots, y_{k}$ of (4.6) the columns of the matrix $\boldsymbol{H}_{1}\left(y_{i}\right)$ are linearly dependent, so for each root $y_{i}$ there exists a non-null vector $\boldsymbol{v}\left(y_{i}\right)$ such that $\boldsymbol{v}\left(y_{i}\right) \boldsymbol{H}_{1}\left(y_{i}\right)=0$. It can be easily shown that the vector $\boldsymbol{v}\left(y_{i}\right)=\left(v_{1}\left(y_{i}\right), v_{2}\left(y_{i}\right), \ldots, v_{k}\left(y_{i}\right)\right)$ has the form

$$
v_{j}\left(y_{i}\right)=\left(\frac{2(k \rho+1) y_{i}-1-\sigma^{2} y_{i}^{2}}{2 k \rho y_{i}}\right)^{j-1} v_{1}\left(y_{i}\right), j=2, \ldots, k,
$$

with $v_{1}\left(y_{i}\right) \neq 0$, for $i=1,2, \ldots, k$. Then from (4.5) we know that the vector $\boldsymbol{Q}(0)$ should be such that $\boldsymbol{v}\left(y_{i}\right) \boldsymbol{H}_{2}\left(y_{i}\right) \boldsymbol{Q}(0)=0$ for all $i=1,2, \ldots, k$. After some calculations we obtain that $\boldsymbol{Q}(0)=$ $\left(q_{1}(0), q_{2}(0), \ldots, q_{k}(0)\right)^{T}$ satisfies the system of linear equations

$$
\left(1-\sigma^{2} y_{i}^{2}\right)\left(\sum_{j=1}^{k} q_{j}(0)\left(\frac{2(k \rho+1) y_{i}-1-\sigma^{2} y_{i}^{2}}{2 k \rho y_{i}}\right)^{j-1}+\frac{2 k \rho}{\sigma^{2}} q_{k}(0)\right)=0
$$


for $i=1,2, \ldots, k$. For non-null solutions of the system (4.11), the system determinant is zero, which can be seen to be equivalent to

$$
\prod_{i=1}^{k}\left(1-\sigma^{2} y_{i}^{2}\right) \prod_{l=1}^{k-1} \prod_{j=l+1}^{k}\left(1-\sigma^{2} y_{l} y_{j}\right)\left(y_{j}-y_{l}\right)=0 .
$$

Assuming that $y_{j}=1 /\left(\sigma^{2} y_{l}\right)$ equation (4.6) implies $y_{l}=y_{j}= \pm 1 / \sigma$, which cannot happen since we have proven that $y_{l} \neq y_{j}$ for all $l \neq j$. Hence, from (4.12) we have that the determinant of the system of equations (4.11) is zero if and only if $1 / \sigma^{2}=y_{k}^{2}$. Then, the linear system for the vector $\boldsymbol{Q}(0)$, after omitting the $k$-th line, assumes the form

$$
\left[\begin{array}{cccc}
v_{1}\left(y_{1}\right) & v_{2}\left(y_{1}\right) & \cdots & v_{k-1}\left(y_{1}\right) \\
v_{1}\left(y_{2}\right) & v_{2}\left(y_{2}\right) & \cdots & v_{k-1}\left(y_{2}\right) \\
\vdots & \vdots & \ddots & \vdots \\
v_{1}\left(y_{k-1}\right) & v_{2}\left(y_{k-1}\right) & \cdots & v_{k-1}\left(y_{k-1}\right)
\end{array}\right]\left[\begin{array}{c}
Q_{0}(0) \\
Q_{1}(0) \\
\vdots \\
Q_{k-2}(0)
\end{array}\right]=\left[\begin{array}{c}
v_{k}\left(y_{1}\right)+\frac{2 k \rho}{\sigma^{2}} v_{1}\left(y_{1}\right) \\
v_{k}\left(y_{2}\right)+\frac{2 k \rho}{\sigma^{2}} v_{1}\left(y_{2}\right) \\
\vdots \\
v_{k}\left(y_{k-1}\right)+\frac{2 k \rho}{\sigma^{2}} v_{1}\left(y_{k-2}\right)
\end{array}\right] Q_{k-1}(0)
$$

with $v_{j}\left(y_{i}\right)$ defined in (4.10). The linear system (4.13) has a non-zero determinant, leading to the conclusion that it has a non-zero solution.

In Lemma 4.2 we have shown that there exists a product-form solution of the equilibrium equations (2.10)-(2.12). Further, Lemma 4.1 implies that this product-form is unique, since the equilibrium distribution of the modified model is unique. Combining Lemmas 4.1 and 4.2 yields the following central result.

Proposition 4.1 For $\rho<1$, the product-form

$$
\boldsymbol{p}(m, n)=\sigma^{2 m} \boldsymbol{q}(n), m, n \geq 0,
$$

with $\sigma$ the unique root of (3.4) inside the open unit circle, and $\boldsymbol{q}(n)=\left(q_{0}(n), \ldots, q_{k-1}(n)\right)^{T}$ non-zero such that $\sum_{n=0}^{\infty} \sigma^{-2 n} q_{h}(n)<\infty, h=0, \ldots, k-1$, is the unique (up to a constant) solution of the equilibrium equations (2.10)-(2.12).

The next results shows why the compensation procedure starts with a product-form satisfying the inner and horizontal axis equilibrium equations, and not with one satisfying the inner and vertical axis equilibrium equations.

Proposition 4.2 For $\rho<1$, there is no $\beta$, with $0<|\beta|<1$, such that the product-form

$$
\boldsymbol{p}(m, n)=\beta^{n} \boldsymbol{q}(m), m, n \geq 0,
$$

with $\boldsymbol{q}(m)=\left(q_{0}(m), \ldots, q_{k-1}(m)\right)^{T}$ non-null such that $\sum_{m=0}^{\infty} q_{h}(m)<\infty, h=0, \ldots, k-1$, is a solution to the equilibrium equations (2.8), (2.9), (2.11) and (2.12).

Proof. Setting $\boldsymbol{p}(m, n)=\beta^{n} \boldsymbol{q}(m)$ in (2.8), (2.9), (2.11) and (2.12) yields two systems of linear difference equations for $\boldsymbol{q}(m)$. Let $\boldsymbol{Q}(y)=\left(Q_{0}(y), \ldots, Q_{k-1}(y)\right)^{T}$, with $Q_{h}(y)=\sum_{m=0}^{\infty} y^{m} q_{h}(m),|y|<1$. We will show that for every $\beta$, with $0<|\beta|<1$, the generating functions $Q_{h}(y), h=0, \ldots, k-1$, are zero for all $y$, with $|y|<1$. 
More concretely, equations (2.9) and (2.11) yields after some calculations the first of the two systems of difference equations

$$
\boldsymbol{H}_{3}(\beta, y) \boldsymbol{Q}(y)=(1-\beta y) \boldsymbol{I}_{k} \boldsymbol{Q}(0)
$$

with

$$
\boldsymbol{H}_{3}(\beta, y)=(-(2 k \rho+2-\beta) \beta y+1) \boldsymbol{I}_{k}+2 k \rho \beta y \boldsymbol{L}_{k}+2 k \rho \beta^{2} y^{2} \boldsymbol{M}_{k}^{(1, k)} .
$$

Furthermore, setting $\boldsymbol{p}(m, n)=\beta^{n} \boldsymbol{q}(m)$ in (2.8) and (2.11) gives the second system of linear difference equations for $\boldsymbol{q}(m)$ :

$$
\boldsymbol{H}_{4}(\beta, y) \boldsymbol{Q}(y)=(2-\beta y) \boldsymbol{I}_{k} \boldsymbol{Q}(0)
$$

with

$$
\boldsymbol{H}_{4}(\beta, y)=\boldsymbol{H}_{3}(\beta, y)+\boldsymbol{I}_{k}+2 k \rho y \boldsymbol{M}_{k}^{(1, k)} .
$$

In light of (4.16) equation (4.17) assumes the form

$$
\left(\boldsymbol{I}_{k}+2 k \rho y \boldsymbol{M}_{k}^{(1, k)}\right) \boldsymbol{Q}(y)=\boldsymbol{I}_{k} \boldsymbol{Q}(0)
$$

which leads to

$$
\boldsymbol{Q}(y)=\left(\boldsymbol{I}_{k}-2 k \rho y \boldsymbol{M}_{k}^{(1, k)}\right) \boldsymbol{I}_{k} \boldsymbol{Q}(0)
$$

Then (4.16) can be written as

$$
\boldsymbol{H}_{5}(\beta, y) \boldsymbol{Q}(0)=0
$$

with

$$
\begin{aligned}
\boldsymbol{H}_{5}(\beta, y) & =\left[\boldsymbol{H}_{3}(\beta, y)\left(\boldsymbol{I}_{k}-2 k \rho y \boldsymbol{M}_{k}^{(1, k)}\right)-(1-\beta y) \boldsymbol{I}_{k}\right] \\
& =\beta y\left[\beta(\beta-1-2 k \rho) \boldsymbol{I}_{k}+2 k \rho \beta \boldsymbol{L}_{k}+2 k \rho(2 \beta y(1+k \rho)-1) \boldsymbol{M}_{k}^{(1, k)}-(2 k \rho)^{2} \beta y \boldsymbol{M}_{k}^{(2, k)}\right] .
\end{aligned}
$$

For $y \neq 0$,

$$
\operatorname{det}\left(\boldsymbol{H}_{5}(\beta, y)\right)=(\beta+1)(2 k \rho \beta)^{k}\left(y-\frac{1}{\beta(\beta+1)}-\frac{1}{\beta+1}\left(1+\frac{1-\beta}{2 k \rho}\right)^{k}\right)
$$

Hence, for $0<|y|<1$ such that $y \neq \frac{1}{\beta(\beta+1)}+\frac{1}{\beta+1}\left(1+\frac{1-\beta}{2 k \rho}\right)^{k}$ the matrix $\boldsymbol{H}_{5}(\beta, y)$ is invertible, so that $\boldsymbol{Q}(0)=\mathbf{0}$, which concludes the proof.

The starting solution obtained in Proposition 4.1 satisfies the inner equilibrium equations as well as the horizontal boundary equations, so we will need to compensate for the error created on the vertical boundary. While performing this compensation the expressions we obtain should always keep satisfying the inner equilibrium equations. For this reason we present Lemmas 4.3 and 4.4 that are needed to describe the form of a solution satisfying the inner equilibrium equations. Furthermore, in Proposition 4.1 we have shown that the starting formula has a unique product-form representation, but we still need to specify the form of the vector $\boldsymbol{q}(n)$. This will be done in Lemma 4.5.

Lemma 4.3 The product-form $\boldsymbol{p}(m, n)=\alpha^{m} \beta^{n} \boldsymbol{\theta}, m \geq 0, n \geq 1$, is a solution of the inner equilibrium equation (2.12) if

$$
\boldsymbol{D}(\alpha, \beta) \boldsymbol{\theta}=0,
$$

where $\boldsymbol{D}(\alpha, \beta)=\alpha \beta \boldsymbol{C}_{0,0}+\alpha \beta^{2} \boldsymbol{A}_{0,-1}+\alpha^{2} \boldsymbol{A}_{-1,1}+\beta^{2} \boldsymbol{A}_{1,-1}$. 


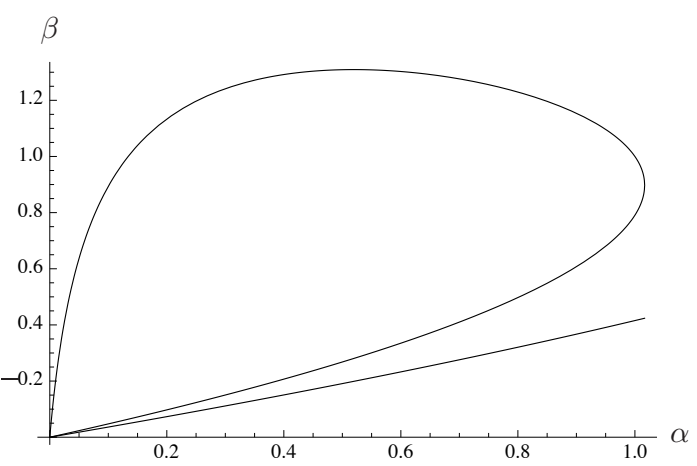

Figure 3: $\operatorname{Det}(\boldsymbol{D}(\alpha, \beta))=0$ in $\mathbb{R}_{+}^{2}$ for $\rho=1 / 8$ and $k=2$.

We should now determine the $\alpha$ 's and $\beta$ 's with $0<|\alpha|<1,0<|\beta|<1$, for which (4.19) has a non-zero solution $\boldsymbol{\theta}$, or equivalently, for which $\operatorname{det}(\boldsymbol{D}(\alpha, \beta))=0$. Fig. 3 depicts the graph of $\operatorname{det}(\boldsymbol{D}(\alpha, \beta))=0$ in $\mathbb{R}_{+}^{2}$ for $\rho=1 / 8$ and $k=2$.

The following lemma provides information about the location and the number of zeros of $\operatorname{det}(\boldsymbol{D}(\alpha, \beta))$.

Lemma 4.4 (i) For every $\alpha$, with $|\alpha| \in(0,1)$, the equation $\operatorname{det}(\boldsymbol{D}(\alpha, \beta))=0$ assumes the form

$$
\left(2(k \rho+1) \beta-\beta^{2}-\alpha\right)^{k} \alpha-(2 k \rho)^{k} \beta^{k+1}=0
$$

and has exactly $k$ simple roots in the $\beta$-plane with $0<|\beta|<|\alpha|$.

(ii) For every $\beta$, with $|\beta| \in(0,1)$, equation (4.20) has exactly one root in the $\alpha$-plane with $0<|\alpha|<|\beta|$.

Proof. (i) Equation (4.20) is a polynomial in $\beta$ of degree $2 k$ and we will show that only $k$ roots are inside the open circle of radius $|\alpha|$. Other possible roots inside the open unit circle appear not to be useful, since they will produce a divergent solution, as we have proved in Proposition 4.1. We divide both sides of equation (4.20) by $\alpha^{k+1}$ and set $z=\beta / \alpha$, which gives

$$
f_{1}^{k}(z)-(2 k \rho)^{k} z^{k+1}=0
$$

with $f_{1}(z)=2(k \rho+1) z-\alpha z^{2}-1$. Observe that (4.21) is equal to (4.7) after setting $z=1 / y$. Hence, according to the proof of Proposition 4.1, the polynomial $f_{1}^{k}(z)-(2 k \rho)^{k} z^{k+1}$ has exactly $k$ roots inside the open unit circle, so we have proven that (4.20) has for each fixed $|\alpha| \in(0,1)$ exactly $k$ roots inside $|\beta|=|\alpha|$. Denote these roots by $\beta_{1}, \beta_{2}, \ldots, \beta_{k}$. We next show that the roots are distinct. To this end, for a root of $g_{1}(z)=f_{1}^{k}(z)-(2 k \rho)^{k} z^{k+1}$ in (4.21) to be of (at least) multiplicity two, it must be that $g_{1}(z)=g_{1}^{\prime}(z)=0$, which gives

$$
\alpha(k-1) z^{2}+2(k \rho+1) z-(k+1)=0 .
$$

Equation (4.22) has two solutions

$$
\hat{z}_{ \pm}=\frac{-(k \rho+1) \pm \sqrt{(k \rho+1)^{2}+\alpha\left(k^{2}-1\right)}}{\alpha(k-1)},
$$

with $\left|\hat{z}_{-}\right|>|\alpha|$ and hence $\beta=\hat{z}_{+}$. By (4.22) we get $\alpha \beta^{2}=(-2(k \rho+1) \beta+k+1) /(k-1)$, so that equation (4.21) can be written as

$$
((k-1) \rho)^{k} \beta=\left(\frac{(k \rho+1) \beta-1}{\beta}\right)^{k} .
$$


Setting $\beta=\hat{z}_{+}$and solving (4.23) in terms of $\alpha$, we will show that all $\alpha$ roots that satisfy the equation exceed the starting solution $\sigma^{2}$, where $\sigma$ is defined as the unique root in $(0,1)$ of $(3.4)$, leading to a contradiction.

Define $u=\sqrt{1+\alpha \frac{k^{2}-1}{(k \rho+1)^{2}}}$. Then either $u \in \mathbb{R}_{+}$or $u \in \mathbb{C} \backslash \mathbb{R}$. Equivalently $\alpha=\frac{u^{2}-1}{k^{2}-1}(k \rho+1)^{2}$ and $\beta=\frac{1}{k \rho+1} \frac{k+1}{1+u}$. Using the form of $\beta$ in (4.23) we obtain after some calculations that

$$
h(u)=(u-k)^{k}(u+1)-(-1)^{k} \frac{\rho^{k}(k+1)^{k+1}(k-1)^{k}}{(k \rho+1)^{k+1}}=0 .
$$

We consider, now two cases for $k=2$ and $k>2$. First, we will treat the case $k>2$. Using the intermediate value theorem the function (4.24) can be easily seen to have at least one real root in the interval $[-\sqrt{2}, 0]$, since $h(0) h(-\sqrt{2})<0$. This root is the only real root of $h(u)$ in the interval $[-\sqrt{2}, \sqrt{2}]$. Assume that there is a second root in the interval $[-\sqrt{2}, 0]$. Then by Rolle's theorem the derivative $h^{\prime}(u)=(k+1)(u-k)^{k-1} u$ should have a real root in the corresponding open interval, which is not the case. Furthermore, the derivative $h^{\prime}(u)=(k+1)(u-k)^{k-1} u$ maintains sign throughout the interval $[0, \sqrt{2}]$ and since $h(0) h(\sqrt{2})>0$ there are no real roots in the interval $[0, \sqrt{2}]$. If we assume that there is one complex root, with $|u|<\sqrt{2}$, then there should be in total at least three roots (the real root, the complex one and its conjugate) inside the circle of radius $\sqrt{2}$, which would mean that there are at least two roots of the derivative $h^{\prime}(u)=(k+1)(u-k)^{k-1} u$ inside the circle of radius $\sqrt{2} / \sin \left(\frac{\pi}{2(k-1)}\right)$ (see e.g. [19]). However one can check that only the zero root is inside the circle since for the other root, $k$ say, one can verify that $k>\sqrt{2} / \sin \left(\frac{\pi}{2(k-1)}\right)$ for all $k>2$.

Hence, there is only one root in the circle of radius $\sqrt{2}$, and this root is a real negative number. All other roots in the $u$-plane will produce $\alpha$-values greater than $\sigma^{2}$ :

$$
|\alpha|=(k \rho+1)^{2} \frac{\left|u^{2}-1\right|}{k^{2}-1} \geq(k \rho+1)^{2} \frac{1}{k^{2}-1}>\rho^{2}>\sigma^{2}, k>2 .
$$

Repeating a similar analysis we observe that for $k=2$ the function (4.24) has exactly one root in the open circle of radius $\sqrt{7} / 2$, and this root is a real negative number. The other two roots in the $u$-plane will produce $\alpha$-values greater than $\sigma^{2}$ :

$$
|\alpha|=(2 \rho+1)^{2} \frac{\left|u^{2}-1\right|}{3} \geq(2 \rho+1)^{2} \frac{1}{4}>\rho^{2}>\sigma^{2} .
$$

(ii) Equation (4.20) is a polynomial in $\alpha$ of degree $k+1$ and we will prove that there is only one root inside the circle of radius $|\beta|$. Dividing both sides of (4.20) by $\beta^{k+1}$ and setting $x=\alpha / \beta$ gives

$$
f_{2}(x)-(2 k \rho)^{k}=0
$$

with $f_{2}(x)=x(2 k \rho+2-\beta-x)^{k}$. Then $f_{2}(x)$ has a zero root of multiplicity one and a root $2 k \rho+2-\beta$ of multiplicity $k$ larger than the radius $3 / 4$. Furthermore, for $|x|=1$, we have that $\left|f_{2}(x)\right| \geq|x|(2 k \rho+2-|\beta|-|x|)^{k}=(2 k \rho+1-|\beta|)^{k}>(2 k \rho)^{k}$, hence by Rouché's theorem $f_{2}(x)-(2 k \rho)^{k}$ has exactly one root in $|x|<1$. This proves that (4.20) has for each fixed $|\beta| \in(0,1)$ exactly one root in $|\alpha|<|\beta|$.

Lemmas 4.3 and 4.4 characterize basic solutions satisfying the inner condition (2.12). In Lemma 4.5 we present the starting solution of the compensation approach. The starting solution was proven in Proposition 4.1 to have a unique product-form representation (up to a positive constant) and in Lemma 4.5 we will specify the form of the vector $\boldsymbol{q}(n)$ appearing in (4.14). 
Lemma 4.5 For $|\alpha| \in(0,1)$ let $\beta_{1}, \beta_{2}, \ldots, \beta_{k}$ be the roots of $(4.20)$ with $|\beta|<|\alpha|$, and let $\boldsymbol{\theta}_{1}, \boldsymbol{\theta}_{2}, \ldots, \boldsymbol{\theta}_{k}$ be the corresponding non-zero vectors satisfying (4.19). Then there exists exactly one $\alpha$ with $0<|\alpha|<1$ for which there are a non-zero vector $\boldsymbol{\xi}$ and coefficients $d_{1}, d_{2}, \ldots, d_{k}$ such that

$$
\boldsymbol{p}(m, n)= \begin{cases}\alpha^{m} \sum_{i=1}^{k} d_{i} \beta_{i}^{n} \boldsymbol{\theta}_{i}, & m \geq 0, n \geq 1 \\ \alpha^{m} \boldsymbol{\xi}, & m \geq 0, n=0\end{cases}
$$

satisfies (2.10)-(2.12). This $\alpha$ is the square of the unique solution of equation (3.4) inside the open unit circle.

The eigenvector $\boldsymbol{\theta}=\left(\theta^{(1)}, \theta^{(2)}, \ldots, \theta^{(k)}\right)^{T}$ satisfies

$$
\frac{\theta^{(i)}}{\theta^{(k)}}=\left(\frac{2(k \rho+1) \beta-\beta^{2}-\alpha}{2 k \rho \beta}\right)^{k-i}, i=1,2, \ldots, k-1 .
$$

The vector $\boldsymbol{\xi}$ is given by

$$
\boldsymbol{\xi}=-\frac{1}{\alpha} \boldsymbol{C}_{0,0}^{-1}\left[\boldsymbol{A}_{0,-1} \alpha+\boldsymbol{A}_{1,-1}\right] \sum_{i=1}^{k} d_{i} \beta_{i} \boldsymbol{\theta}_{i}
$$

and the coefficients $d_{1}, d_{2}, \ldots, d_{k}$ satisfy

$$
\sum_{j=1}^{k} \boldsymbol{K}\left(\alpha, \beta_{j}\right) d_{j}=0
$$

with $\boldsymbol{K}\left(\alpha, \beta_{j}\right)=\left(K_{1}\left(\alpha, \beta_{j}\right), \ldots, K_{k}\left(\alpha, \beta_{j}\right)\right)^{T}$

$$
\begin{aligned}
& K_{1}\left(\alpha, \beta_{j}\right)=\beta_{j}\left(\frac{\alpha}{\beta_{j}^{2}+\alpha}\left(\frac{k \rho}{k \rho+1}\right)^{k}+\frac{\alpha}{2(k \rho+1)} \frac{2(k \rho+1) \beta_{j}-2 \alpha}{2(k \rho+1) \beta_{j}-\beta_{j}^{2}-\alpha}+\frac{\alpha \beta_{j}}{\beta_{j}^{2}+\alpha}\right) \theta_{j}^{(k)}, \\
& K_{i}\left(\alpha, \beta_{j}\right)=\frac{\beta_{j}}{\beta_{j}^{2}+\alpha}\left(2 \alpha\left(\frac{k \rho}{k \rho+1}\right)^{i}+\left(\beta_{j}^{2}-\alpha\right)\left(\frac{2 k \rho \beta_{j}}{2(k \rho+1) \beta_{j}-\beta_{j}^{2}-\alpha}\right)^{i}\right) \theta_{j}^{(k)},
\end{aligned}
$$

for $i=2, \ldots, k, j=1, \ldots, k$.

Proof. Substituting in (2.10) the expression for $\boldsymbol{p}(m, n)$ in (4.26) immediately yields (4.28). Then, from (2.11) we obtain

$$
\sum_{i=1}^{k} d_{i} \beta_{i}\left[\boldsymbol{C}_{0,0} \alpha+\boldsymbol{A}_{0,-1} \alpha \beta_{i}+\boldsymbol{A}_{1,-1} \beta_{i}-\left[2 \boldsymbol{A}_{-1,1} \alpha+\boldsymbol{A}_{0,1}\right] \boldsymbol{C}_{0,0}^{-1}\left[\boldsymbol{A}_{0,-1} \alpha+\boldsymbol{A}_{1,-1}\right]\right] \boldsymbol{\theta}_{i}=\mathbf{0}
$$

After tedious calculations the above matrix expression can be simplified to expression (4.29).

Remark 4.1 The rank of matrix $\boldsymbol{K}=\left[\boldsymbol{K}\left(\alpha, \beta_{1}\right) \ldots \boldsymbol{K}\left(\alpha, \beta_{k}\right)\right]$ in (4.29) is equal to $k-1$. This can be proven as follows. First notice that $\operatorname{rank}(\boldsymbol{K})<k$, since the equilibrium distribution of the modified model is non-zero under stability. Further, if $\operatorname{rank}(\boldsymbol{K})<k-1$, then there exist at least two linearly independent solutions $\boldsymbol{d}_{1}$ and $\boldsymbol{d}_{2}$, hence there would be at least two linearly independent vectors $\boldsymbol{q}_{1}(m)$ and $\boldsymbol{q}_{2}(m)$ satisfying (4.1). This contradicts the uniqueness of the equilibrium distribution of the modified model. 
The starting formula in the upper plane is a linear combination of $k$ terms. One can easily check that these terms do not satisfy the vertical boundary. Let us consider a term $d \alpha^{m} \beta^{n} \boldsymbol{\theta}$ (with $|\beta|<|\alpha|$ ) and show how to compensate for the error of this term on the vertical boundary. The idea behind the compensation approach is to add a new term $c \hat{\alpha}^{m} \hat{\beta}^{n} \hat{\boldsymbol{\theta}}$, such that $d \alpha^{m} \beta^{n} \boldsymbol{\theta}+c \hat{\alpha}^{m} \hat{\beta}^{n} \hat{\boldsymbol{\theta}}$ satisfies both (2.9) and (2.12). Substituting this linear combination into (2.9) yields a set of $k$ equations of the form

$$
d \beta^{n-1} \boldsymbol{H}(\alpha, \beta) \boldsymbol{\theta}+c \hat{\beta}^{n-1} \boldsymbol{H}(\hat{\alpha}, \hat{\beta}) \hat{\boldsymbol{\theta}}=\mathbf{0}, n>1,
$$

where $\boldsymbol{H}(x, y)=y \boldsymbol{B}_{0,0}+y^{2} \boldsymbol{A}_{1,0}+x \boldsymbol{A}_{0,-1}$. Hence, $\beta=\hat{\beta}$, and therefore $\hat{\alpha}$ can be obtained from (4.20) and $\hat{\boldsymbol{\theta}}$ is the corresponding non-zero solution of (4.19), only leaving $c$ for meeting $k$ requirements. Thus the choice of $d \alpha^{m} \beta^{n} \boldsymbol{\theta}+c \hat{\alpha}^{m} \hat{\beta}^{n} \hat{\boldsymbol{\theta}}$ for $m, n \geq 0$ does not provide enough freedom to satisfy the boundary equations. Remarkably, the constant $c$, as we will prove in Lemma 4.6, is very close to our initial approach of simply adding the term $c \hat{\alpha}^{m} \beta^{n} \hat{\boldsymbol{\theta}}$.

We shall next describe the compensation on the vertical boundary.

Lemma 4.6 For $|\beta| \in(0,1)$ let $\alpha, \hat{\alpha}$ be roots of (4.20)) with $|\hat{\alpha}|<|\beta|<|\alpha|$, and let $\boldsymbol{\theta}, \hat{\boldsymbol{\theta}}$ be the corresponding non-zero vectors satisfying (4.19). Then there exists a coefficient $c$ such that

$$
\boldsymbol{p}(m, n)= \begin{cases}d \alpha^{m} \beta^{n} \boldsymbol{\theta}+c \hat{\alpha}^{m} \beta^{n} \hat{\boldsymbol{\theta}}, & m>0, n \geq 0, \\ d \frac{\alpha}{\alpha-\beta} \beta^{n} \boldsymbol{\theta}+c \frac{\hat{\alpha}}{\hat{\alpha}-\beta} \beta^{n} \hat{\boldsymbol{\theta}}, & m=0, n \geq 0,\end{cases}
$$

satisfy both (2.9) and (2.12). The coefficient $c$ is given by

$$
c=d \frac{\beta-\hat{\alpha}}{\alpha-\beta} \frac{\theta^{(k)}}{\hat{\theta}^{(k)}}
$$

The eigenvector $\boldsymbol{\theta}$ is given in (4.27) and $\hat{\boldsymbol{\theta}}=\left(\hat{\theta}^{(1)}, \hat{\theta}^{(2)}, \ldots, \hat{\theta}^{(k)}\right)^{T}$ satisfies

$$
\frac{\hat{\theta}^{(i)}}{\hat{\theta}^{(k)}}=\left(\frac{2(k \rho+1) \beta-\beta^{2}-\hat{\alpha}}{2 k \rho \beta}\right)^{k-i}, i=1,2, \ldots, k-1 .
$$

Proof. We observe that the equilibrium equations (2.4)-(2.6) for $m=1$ demand that the equilibrium distribution $p(k-1,0, n)$, for $n \geq 1$, is of the same geometric form as $p(h, m, n), m \geq 1$. Hence, we need only to assume that

$$
p(k-1,0, n)=\left(d \theta^{(k)}+c \hat{\theta}^{(k)}\right) \beta^{n} .
$$

We will determine $\boldsymbol{p}(0, n)$ iteratively through (2.3). Because of the form of equation (2.3) we can assume that

$$
p(h, 0, n)=\left(d p_{h+1} \theta^{(h+1)}+c \hat{p}_{h+1} \hat{\theta}^{(h+1)}\right) \beta^{n}, h=0, \ldots, k-1 .
$$

Then plugging equation (4.36) into equation (2.3) for $h=1,2, \ldots, k-1$ and using (4.27) we have that the sequences $p_{h+1}$ and $\hat{p}_{h+1}$ satisfy the recursive schemes $p_{h}=\eta\left(p_{h+1}\right)$ and $\hat{p}_{h}=\hat{\eta}\left(\hat{p}_{h+1}\right)$, for $h=0,1, \ldots, k-1$ and

$$
\eta(x)=\frac{(2 k \rho+1) \beta x-\beta^{2} x-\alpha}{2(k \rho+1) \beta-\beta^{2}-\alpha}, \hat{\eta}(x)=\frac{(2 k \rho+1) \beta x-\beta^{2} x-\hat{\alpha}}{2(k \rho+1) \beta-\beta^{2}-\hat{\alpha}}
$$


with initial conditions $p_{k}=\hat{p}_{k}=1$. Solving these linear difference equations gives for $h=0, \ldots, k-1$

$$
\frac{p(h, 0, n)}{\beta^{n}}=d \frac{\alpha-\beta\left(\frac{(2 k \rho+1) \beta-\beta^{2}}{2(k \rho+1) \beta-\beta^{2}-\alpha}\right)^{k-h-1}}{\alpha-\beta} \theta^{(h+1)}+c \frac{\hat{\alpha}-\beta\left(\frac{(2 k \rho+1) \beta-\beta^{2}}{2(k \rho+1) \beta-\beta^{2}-\hat{\alpha}}\right)^{k-h-1}}{\hat{\alpha}-\beta} \hat{\theta}^{(h+1)} .
$$

Equation (2.3) for $h=0$ yields

$$
c=-d \frac{(2 k \rho+1) \beta p_{1}-\beta^{2} p_{1}-\alpha}{(2 k \rho+1) \beta \hat{p}_{1}-\beta^{2} \hat{p}_{1}-\hat{\alpha}} \frac{\theta^{(1)}}{\hat{\theta}^{(1)}} .
$$

Using the recursive scheme for the sequences $p_{h+1}$ and $\hat{p}_{h+1}$ and (4.37) gives

$$
\begin{aligned}
c & =-d \frac{p_{0}}{\hat{p}_{0}} \frac{2(k \rho+1) \beta-\beta^{2}-\alpha}{2(k \rho+1) \beta-\beta^{2}-\hat{\alpha}} \frac{\theta^{(1)}}{\hat{\theta}^{(1)}} \\
& =-d \frac{\frac{\alpha-\beta\left(\frac{(2 k \rho+1) \beta-\beta^{2}}{2(k \rho+1) \beta-\beta^{2}-\alpha}\right)^{k}}{\alpha-\beta}}{\frac{\hat{\alpha}-\beta\left(\frac{(2 k \rho+1) \beta-\beta^{2}}{2(k \rho+1) \beta-\beta^{2}-\hat{\alpha}}\right)^{k}}{\hat{\alpha}-\beta}} \frac{2(k \rho+1) \beta-\beta^{2}-\alpha}{2(k \rho+1) \beta-\beta^{2}-\hat{\alpha}} \frac{\theta^{(1)}}{\hat{\theta}^{(1)}} .
\end{aligned}
$$

Using (4.20) and (4.27) gives

$$
\begin{aligned}
c & =-d \frac{\frac{\alpha-\alpha\left(\frac{2 k \rho+1-\beta}{2 k \rho}\right)^{k}}{\alpha-\beta}}{\frac{\hat{\alpha}-\hat{\alpha}\left(\frac{2 k \rho+1-\beta}{2 k \rho}\right)^{k}}{\hat{\alpha}-\beta}} \frac{2(k \rho+1) \beta-\beta^{2}-\alpha}{2(k \rho+1) \beta-\beta^{2}-\hat{\alpha}} \frac{\theta^{(1)}}{\hat{\theta}^{(1)}} \\
& =d \frac{\beta-\hat{\alpha}}{\alpha-\beta} \frac{\alpha\left(2(k \rho+1) \beta-\beta^{2}-\alpha\right)^{k}}{\hat{\alpha}\left(2(k \rho+1) \beta-\beta^{2}-\hat{\alpha}\right)^{k}} \frac{\theta^{(k)}}{\hat{\theta}^{(k)}} \\
& =d \frac{\beta-\hat{\alpha}}{\alpha-\beta} \frac{\theta^{(k)}}{\hat{\theta}^{(k)}} .
\end{aligned}
$$

In light of equation (4.33) equation (4.37) can be simplified, after eliminating the common terms, to (4.32).

Compensation on the vertical boundary has created an error and one can check that the new fromula does not satisfy the horizontal boundary. Let us consider the term $c \hat{\alpha}^{m} \beta^{n} \hat{\boldsymbol{\theta}}$ (with $|\hat{\alpha}|<|\beta|$ ) and show how to compensate for the error of this term on the horizontal boundary. We will add a new term $\check{\alpha}^{m} \sum_{i=1}^{k} \hat{d}_{i} \hat{\beta}^{n} \check{\boldsymbol{\theta}}$, such that $c \hat{\alpha}^{m} \beta^{n} \hat{\boldsymbol{\theta}}+\check{\alpha}^{m} \sum_{i=1}^{k} \hat{d}_{i} \hat{\beta}^{n} \check{\boldsymbol{\theta}}$ satisfies (2.10)-(2.12). Substituting this linear combination into (2.10) and (2.11) yields a set of $k$ equations of the form

$$
c \hat{\alpha}^{m-1} \beta \boldsymbol{G}(\hat{\alpha}, \beta) \boldsymbol{\theta}+\check{\alpha}^{m-1} \sum_{i=1}^{k} \hat{d}_{i} \hat{\beta}_{i} \boldsymbol{G}\left(\check{\alpha}, \hat{\beta}_{i}\right) \hat{\boldsymbol{\theta}}_{i}=\mathbf{0}, m>1,
$$

where $\boldsymbol{G}(x, y)=x \boldsymbol{C}_{0,0}+x y \boldsymbol{A}_{0,-1}+y \boldsymbol{A}_{1,-1}-\left[2 x \boldsymbol{A}_{-1,1}+\boldsymbol{A}_{0,1}\right] \boldsymbol{C}_{0,0}^{-1}\left[x \boldsymbol{A}_{0,-1}+\boldsymbol{A}_{1,-1}\right]$. Hence, $\hat{\alpha}=\check{\alpha}$, and therefore $\hat{\beta}_{1}, \ldots, \hat{\beta}_{k}$ can be obtained from (4.20) and $\check{\boldsymbol{\theta}}_{1}, \ldots, \check{\boldsymbol{\theta}}_{k}$ are the corresponding non-zero solutions of (4.19), only leaving $\hat{d}_{1}, \ldots, \hat{d}_{k}$ to be calculated from the set of the $k$ equations.

The next result presents the compensation on the horizontal boundary. 
Lemma 4.7 For $|\hat{\alpha}| \in(0,1)$ let $\beta, \hat{\beta}_{1}, \hat{\beta}_{2}, \ldots, \hat{\beta}_{k}$ be the roots of $(4.20)$ with $|\hat{\beta}|<|\hat{\alpha}|<|\beta|$, and let $\hat{\boldsymbol{\theta}}, \check{\boldsymbol{\theta}}_{1}, \check{\boldsymbol{\theta}}_{2}, \ldots, \check{\boldsymbol{\theta}}_{k}$ be the corresponding non-zero vectors satisfying (4.19). Then there exists a non-zero vector $\hat{\boldsymbol{\xi}}$ and coefficients $\hat{d}_{1}, \hat{d}_{2}, \ldots, \hat{d}_{k}$ such that

$$
\boldsymbol{p}(m, n)= \begin{cases}c \hat{\alpha}^{m} \beta^{n} \hat{\boldsymbol{\theta}}+\hat{\alpha}^{m} \sum_{i=1}^{k} \hat{d}_{i} \hat{\beta}_{i}{ }^{n} \check{\boldsymbol{\theta}}_{i}, & m \geq 0, n \geq 1, \\ \alpha^{m} \boldsymbol{\xi}+\hat{\alpha}^{m} \hat{\boldsymbol{\xi}} & m \geq 0, n=0,\end{cases}
$$

satisfy (2.10)-(2.12). The eigenvector $\hat{\boldsymbol{\theta}}$ is given in $(4.34)$ and $\check{\boldsymbol{\theta}}=\left(\check{\theta}^{(1)}, \check{\theta}^{(2)}, \ldots, \check{\theta}^{(k)}\right)^{T}$ satisfies

$$
\frac{\check{\theta}^{(i)}}{\check{\theta}^{(k)}}=\left(\frac{2(k \rho+1) \hat{\beta}-\hat{\beta}^{2}-\hat{\alpha}}{2 k \rho \hat{\beta}}\right)^{k-i}, i=1,2, \ldots, k-1 .
$$

The vector $\hat{\boldsymbol{\xi}}$ is given by

$$
\hat{\boldsymbol{\xi}}=-\boldsymbol{\xi}-\frac{1}{\hat{\alpha}} \boldsymbol{C}_{0,0}^{-1}\left[\boldsymbol{A}_{0,-1} \hat{\alpha}+\boldsymbol{A}_{1,-1}\right]\left(c \beta \hat{\boldsymbol{\theta}}+\sum_{i=1}^{k} \hat{d}_{i} \hat{\beta}_{i} \check{\boldsymbol{\theta}}_{i}\right),
$$

where $\boldsymbol{\xi}$ is given in (4.28). The coefficients $\hat{d}_{1}, \hat{d}_{2}, \ldots, \hat{d}_{k}$ satisfy

$$
\sum_{j=1}^{k} \boldsymbol{K}\left(\hat{\alpha}, \hat{\beta}_{j}\right) \hat{d}_{j}=-c \boldsymbol{K}(\hat{\alpha}, \beta)
$$

with $\boldsymbol{K}(\alpha, \beta)=\left(K_{1}(\alpha, \beta), \ldots, K_{k}(\alpha, \beta)\right)^{T}$ defined in (4.30) and (4.31).

Proof. The proof is identical to the proof of Lemma 4.5.

Repeating the compensation procedure by adding terms so as to compensate alternatively for the error on the boundary $m=0$ according to Lemma 4.6, and on the boundary $n=0$ according to Lemma 4.7 , will generate a sequence of terms. These sequences of compensation terms grow as a $k$-ary tree and the problem is to prove that they converge. First, we will describe the compensation procedure, then we will formally define the final expression in terms of infinite series and afterwards prove the convergence.

\section{Description of the compensation approach}

The compensation approach will lead to an expression for $\boldsymbol{p}(m, n)$ in terms of an infinite series of product-forms. We next describe how the product-forms can be obtained in an iterative fashion.

$H_{0}$ : Start with $\alpha_{0,1}$, the unique root of equation (3.4) inside the open unit circle, and calculate the $k \beta$ 's from Lemma 4.4 , denoted by $\beta_{0,1}, \beta_{0,2}, \ldots, \beta_{0, k}$. The corresponding non-zero $\boldsymbol{\theta}$ vectors and the coefficients $d$ from Lemma 4.3 are denoted by $\boldsymbol{\theta}_{0,1}, \boldsymbol{\theta}_{0,2}, \ldots, \boldsymbol{\theta}_{0, k}$ and $d_{0,1}, d_{0,2}, \ldots, d_{0, k}$, respectively. This gives the product-form $\alpha_{0,1}^{m} \sum_{j=1}^{k} d_{0, j} \beta_{0, j}^{n} \boldsymbol{\theta}_{0, j}$.

$V_{0}$ : For $\beta_{0, j}, j=1,2, \ldots, k$, calculate the $\alpha$ 's from Lemma 4.4, denoted by $\alpha_{1, j}$. The corresponding non-zero $\hat{\boldsymbol{\theta}}$ vectors and the coefficients $c$ from Lemma 4.6 are denoted by $\hat{\boldsymbol{\theta}}_{1, j}$ and $c_{0, j}$, respectively. This gives the product-form $\sum_{j=1}^{k} c_{0, j} \alpha_{1, j}^{m} \beta_{0, j}^{n} \hat{\boldsymbol{\theta}}_{1, j}$. 
$H_{1}$ : For $\alpha_{1, i}, i=1,2, \ldots, k$, obtained in the previous step, calculate the $k$ 's from Lemma 4.4 , denoted by $\beta_{1, k i-j}, j=0, \ldots, k-1$. The corresponding non-zero $\check{\boldsymbol{\theta}}$ vectors and the coefficients $\hat{d}$ from Lemma 4.7 are denoted by $\boldsymbol{\theta}_{1, k i-j}$ and $d_{1, k i-j}$, respectively. This gives the product-form $\sum_{i=1}^{k^{2}} \alpha_{1, i}^{m} \sum_{j=0}^{k-1} d_{1, k i-j} \beta_{1, k i-j}^{n} \boldsymbol{\theta}_{1, k i-j}$.

$V_{1}$ : For $\beta_{1, j}, j=1,2, \ldots, k^{2}$, calculate the $\alpha$ 's from Lemma 4.4 , denoted by $\alpha_{2, j}$. The corresponding non-zero $\hat{\boldsymbol{\theta}}$ vectors and the coefficients $c$ from Lemma 4.6 are denoted by $\hat{\boldsymbol{\theta}}_{2, j}$ and $c_{1, j}$, respectively. This gives the product-form $\sum_{j=1}^{k^{2}} c_{1, j} \alpha_{2, j}^{m} \beta_{1, j}^{n} \hat{\boldsymbol{\theta}}_{2, j}$.

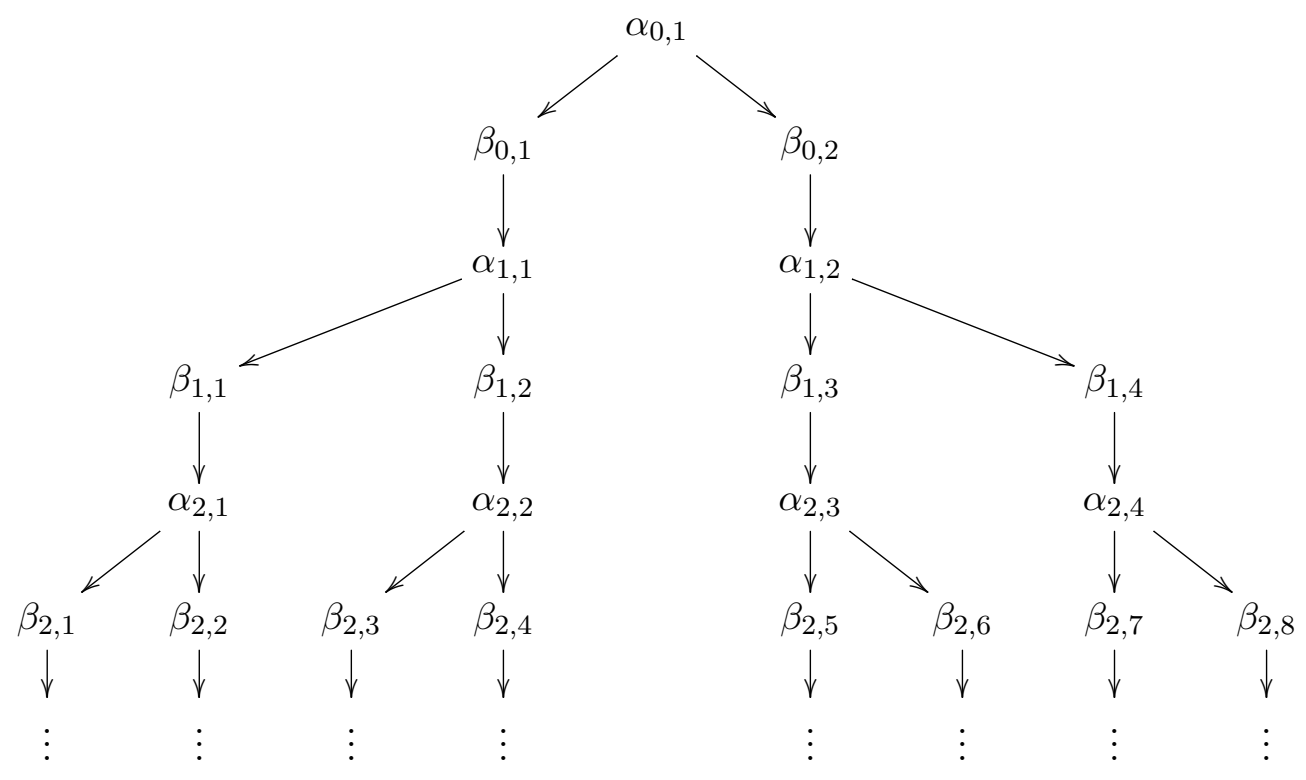

Figure 4: Tree structure of $\alpha$ 's and $\beta$ 's for $k=2$

A general step of this process is described as follows:

$H_{\ell}$ : For $\alpha_{\ell, i}, i=1,2, \ldots, k^{\ell}$, calculate the $k \beta$ 's from Lemma 4.4 , denoted by $\beta_{\ell, k i-j}, j=0, \ldots, k-1$. The corresponding non-zero $\check{\boldsymbol{\theta}}$ vectors and the coefficients $\hat{d}$ from Lemma 4.7 are denoted by $\boldsymbol{\theta}_{\ell, k i-j}$ and $d_{\ell, k i-j}$, respectively. This gives the product-form $\sum_{i=1}^{k^{\ell}} \alpha_{\ell, i}^{m} \sum_{j=0}^{k-1} d_{\ell, k i-j} \beta_{\ell, k i-j}^{n} \boldsymbol{\theta}_{\ell, k i-j}$.

$V_{\ell}:$ For $\beta_{\ell, j}, j=1,2, \ldots, k^{\ell+1}$, calculate the $\alpha$ from Lemma 4.4 , denoted by $\alpha_{\ell+1, j}$. The corresponding non-zero $\hat{\boldsymbol{\theta}}$ vectors and the coefficients $c$ from Lemma 4.6 are denoted by $\hat{\boldsymbol{\theta}}_{\ell+1, j}$ and $c_{\ell, j}$. This gives the product-form $\sum_{j=1}^{k^{\ell+1}} c_{\ell, j} \alpha_{\ell+1, j}^{m} \beta_{\ell, j}^{n} \hat{\boldsymbol{\theta}}_{\ell+1, j}$.

Continuing the above procedure leads to all pairs of $\alpha$ and $\beta$ for which the corresponding vectors $\boldsymbol{\theta}$ or $\hat{\boldsymbol{\theta}}$ and the coefficients $d$ or $\hat{d}$ and $c$ are all the basic solutions for constructing a formal expression for the equilibrium probability vectors $\boldsymbol{p}(m, n)$. The tree structure of consecutive $\alpha$ 's and $\beta$ 's is illustrated in Fig. 4.

The compensation approach eventually leads to the following expression for the equilibrium distribution. 
Theorem 5.1 For all states $(h, m, n), h=0,1, \ldots, k-1$,

$$
\begin{aligned}
\boldsymbol{p}(m, n) & \propto \sum_{\ell=0}^{\infty} \sum_{i=1}^{k^{\ell}} \alpha_{\ell, i}^{m} \sum_{j=0}^{k-1} d_{\ell, k i-j} \beta_{\ell, k i-j}^{n} \boldsymbol{\theta}_{\ell, k i-j} \\
& +\sum_{\ell=0}^{\infty} \sum_{i=1}^{k^{\ell+1}} c_{\ell, i} \alpha_{\ell+1, i}^{m} \beta_{\ell, i}^{n} \hat{\boldsymbol{\theta}}_{\ell+1, i}, m>0, n>0, \\
\boldsymbol{p}(m, 0) \propto & \sum_{\ell=0}^{\infty} \sum_{i=1}^{k^{\ell}} \alpha_{\ell, i}^{m} \boldsymbol{\xi}_{\ell, i}, m>0, \\
\boldsymbol{p}(0, n) \propto & \sum_{\ell=0}^{\infty} \sum_{i=1}^{k^{\ell}} \sum_{j=0}^{k-1} \frac{\alpha_{\ell, i}}{\beta_{\ell, k i-j}-\alpha_{\ell, i}} d_{\ell, k i-j} \beta_{\ell, k i-j}^{n} \boldsymbol{\theta}_{\ell, k i-j} \\
& +\sum_{\ell=0}^{\infty} \sum_{i=1}^{k^{\ell+1}} \frac{\alpha_{\ell+1, i}}{\beta_{\ell, i}-\alpha_{\ell+1, i}} c_{\ell, i} \beta_{\ell, i}^{n} \hat{\boldsymbol{\theta}}_{\ell+1, i}, n>0, \\
\boldsymbol{p}(0,0) \propto & \frac{1}{2 k \rho} \boldsymbol{T}_{k} \boldsymbol{p}(0,1),
\end{aligned}
$$

where $\boldsymbol{T}_{k}$ denotes a $k \times k$ lower triangular matrix, with elements $(i, j), i \geq j, j=1, \ldots, k$, equal to one and zeros elsewhere.

There remains the task of proving that these formal expressions are in fact absolutely convergent. However, before doing so we need several preliminary results. The analysis that will follow is similar to the analysis performed in [4]. First, we analyze the limiting behavior of the sequences $\left\{\alpha_{\ell, i}\right\}_{\ell \in \mathbb{N}_{0}, i=1, \ldots, k^{\ell}}$ and $\left\{\beta_{\ell, i}\right\}_{\ell \in \mathbb{N}_{0}, i=1, \ldots, k^{\ell+1}}$ and the corresponding sequences of coefficients. The following proposition provides geometric bounds for the $\alpha$ 's and $\beta$ 's.

Proposition 5.1 The $\left\{\alpha_{\ell, i}\right\}_{\ell \in \mathbb{N}_{0}, i=1, \ldots, k^{\ell}}$ and $\left\{\beta_{\ell, i}\right\}_{\ell \in \mathbb{N}_{0}, i=1, \ldots, k^{\ell+1}}$ appearing in (5.1)-(5.4) satisfy the properties

(i) $1 \geq\left|\alpha_{0,1}\right|>\max \left\{\left|\beta_{0,1}\right|, \ldots,\left|\beta_{0, k}\right|\right\}>\max \left\{\left|\alpha_{1,1}\right|, \ldots,\left|\alpha_{1, k}\right|\right\}>\max \left\{\left|\beta_{1,1}\right|, \ldots,\left|\beta_{1, k^{2}}\right|\right\}>\ldots$

(ii) $0 \leq \max \left\{\left|\alpha_{\ell, 1}\right|, \ldots,\left|\alpha_{\ell, k^{\ell}}\right|\right\} \leq\left(\frac{3}{8}\right)^{\ell}$ and $0 \leq \max \left\{\left|\beta_{\ell, 1}\right|, \ldots,\left|\beta_{\ell, k^{\ell+1}}\right|\right\} \leq \frac{1}{2}\left(\frac{3}{8}\right)^{\ell}$.

Proof. (i) Remember that $\alpha_{0,1}$ is the root of equation (3.4) inside the open unit circle, hence $\left|\alpha_{0,1}\right| \leq 1$, for all $\rho \in(0,1)$. Then, $\beta_{0,1}, \ldots, \beta_{0, k}$ follow from (4.20) and according to Lemma 4.4 $\max \left\{\left|\beta_{0,1}\right|, \ldots,\left|\beta_{0, k}\right|\right\}<\left|\alpha_{0,1}\right|$, so that assertion (i) follows upon repeating this argument.

(ii) We only need to prove that for each $\alpha$, with $|\alpha|<1$, we obtain $k \beta$ 's with $|\beta|<|\alpha| / 2$ and that for each $\beta$, with $|\beta|<1$, we obtain one $\alpha$ with $|\alpha|<3|\beta| / 4$. These two proofs are almost identical to the proofs of Lemma 4.4 and for this reason we omit them (see Appendix C).

It follows from Proposition 5.1 that the sequences of $\left\{\alpha_{\ell, i}\right\}$ and $\left\{\beta_{\ell, i}\right\}$ tend to zero as $\ell$ tends to infinity. The limiting behavior of the sequences of $\alpha$ 's and $\beta$ 's is presented in Lemma 5.1 and the limiting behavior of the coefficients is treated in Lemma 5.2 . 


\section{Lemma 5.1}

(i) Let $\alpha, \beta$ and $\hat{\alpha}$ be roots of equation (4.20) for fixed $\alpha$, with $1>|\alpha|>|\beta|>|\hat{\alpha}|$. Then, as $\alpha \rightarrow 0$, $\beta / \alpha \rightarrow w$, with $|w|<1$, and $\hat{\alpha} / \beta \rightarrow 1 / \hat{w}$, with $|\hat{w}|>1$, and both $w$ and $\hat{w}$ are roots of

$$
(2(k \rho+1) w-1)^{k}=(2 k \rho)^{k} w^{k+1} .
$$

(ii) Let $\beta$, $\hat{\alpha}$ and $\hat{\beta}$ be roots of equation (4.20) for fixed $\beta$, with $1>|\beta|>|\hat{\alpha}|>|\hat{\beta}|$. Then, as $\beta \rightarrow 0$, $\hat{\beta} / \hat{\alpha} \rightarrow w$, with $|w|<1$, and $\hat{\alpha} / \beta \rightarrow 1 / \hat{w}$, with $|\hat{w}|>1$, and both $w$ and $\hat{w}$ are roots of (5.5).

Corollary 5.1 The equation (5.5) has $k$ roots inside the open unit circle, denoted by $w_{1}, \ldots, w_{k}$, and a real root outside denoted by $w_{k+1}$. Furthermore, $w_{1}, \ldots, w_{k+1}$ roots have the following characteristics:

(i) $w_{1}, \ldots, w_{k}$ are distinct and $\left|w_{i}\right|<1 / 2$ for $i=1, \ldots, k$.

$$
\frac{1}{2}\left(1+\frac{1}{k \rho}\right)^{k}<w_{k+1}
$$

Proof. We rewrite (5.5) as

$$
f_{3}^{k}(w)-(2 k \rho)^{k} w^{k+1}=0
$$

with $f_{3}(w)=2(k \rho+1) w-1$, and we observe that $f_{3}(w)$ has a single root $w=\frac{1}{2(k \rho+1)}<\frac{1}{2}$. Furthermore, for $|w|=1$ we have that $\left|f_{3}(w)\right|^{k} \geq|2(k \rho+1)| w|-1|^{k}=(2 k \rho+1)^{k}>(2 k \rho)^{k}$, hence by Rouché's theorem $f_{3}^{k}(w)-(2 k \rho)^{k} w^{k+1}$ has exactly $k$ roots in $|w|<1$ and one root in $|w| \geq 1$. The root outside the open unit circle can be easily verified to be a real root by applying the intermediate value theorem on the interval $[1, \infty)$.

(i) Again using Rouché's theorem on $|w|=\frac{1}{2}$ leads to the more strict bound for the roots inside the open unit circle as stated in assertion (ii). For a root of $g_{3}(w)=f_{3}^{k}(w)-(2 k \rho)^{k} w^{k+1}$ to be of (at least) multiplicity two, it must be that $g_{3}(w)=g_{3}^{\prime}(w)=0$, which gives $w=\frac{1}{2} \frac{k+1}{k \rho+1}>\frac{1}{2}$. Hence all roots $w_{1}, \ldots, w_{k}$ inside the open circle are distinct.

(ii) Proving that $w_{k+1}>\frac{1}{2}\left(1+\frac{1}{k \rho}\right)^{k}$ is equivalent with showing that inside the circle of radius $|w|=$ $\frac{1}{2}\left(1+\frac{1}{k \rho}\right)^{k}$ the polynomial $f_{3}^{k}(w)-(2 k \rho)^{k} w^{k+1}=0$ has exactly $k$ roots. This proof is identical to the proof that the polynomial has $k$ roots inside the open unit circle and for this reason is omitted.

\section{Lemma 5.2}

(i) Let $\alpha$ and $\beta_{1}, \ldots, \beta_{k}$ be roots of equation (4.20) for fixed $\alpha$, with $1>|\alpha|>\max \left\{\left|\beta_{1}\right|, \ldots,\left|\beta_{k}\right|\right\}$. Then, as $\alpha \rightarrow 0$,

(a) The vector $\boldsymbol{\theta}_{i}=\left(\theta_{i}^{(1)}, \ldots, \theta_{i}^{(k)}\right)^{T}$ converges to

$$
\frac{\theta_{i}^{(h)}}{\theta_{i}^{(k)}} \rightarrow\left(\frac{2(k \rho+1) w_{i}-1}{2 k \rho w_{i}}\right)^{k-h}, h=1,2, \ldots, k-1,
$$

where $w_{1}, \ldots, w_{k}$ are the roots of (5.5) inside the open unit circle. 
(b) The system (4.29) for determining the coefficients $d_{1}, \ldots, d_{k}$ reduces to

$$
\sum_{j=1}^{k} \boldsymbol{\theta}_{j} d_{j}=\mathbf{0}
$$

and can be explicitly solved as

$$
\frac{d_{j}}{d_{k}}=-\prod_{\substack{i=1 \\ i \neq j}}^{k-1} \frac{w_{i}-w_{k}}{w_{i}-w_{j}}, j=1, \ldots, k-1
$$

(c) The vector $\boldsymbol{\xi}$ converges to

$$
\boldsymbol{\xi}=-\boldsymbol{C}_{0,0}^{-1} \boldsymbol{A}_{1,-1} \sum_{j=1}^{k} d_{j} w_{j} \boldsymbol{\theta}_{j}
$$

(ii) Let $\beta_{i}$ and $\hat{\alpha}$ be roots of equation (4.20) for fixed $\beta_{i}$, with $1>\left|\beta_{i}\right|>|\hat{\alpha}|, i=1, \ldots, k$. Then, as $\beta_{i} \rightarrow 0$,

(a) The vector $\hat{\boldsymbol{\theta}}=\left(\hat{\theta}^{(1)}, \ldots, \hat{\theta}^{(k)}\right)^{T}$ converges to

$$
\frac{\hat{\theta}^{(h)}}{\hat{\theta}^{(k)}} \rightarrow\left(\frac{2(k \rho+1) w_{k+1}-1}{2 k \rho w_{k+1}}\right)^{k-h}, h=1,2, \ldots, k-1,
$$

where $w_{k+1}$ is the root of (5.5) outside the open unit circle.

(b) The coefficient $c / d_{i}$ converges to

$$
c / d_{i} \rightarrow \frac{w_{i}}{w_{k+1}} \frac{w_{k+1}-1}{1-w_{i}} \frac{\theta_{i}^{(k)}}{\hat{\theta}_{i}^{(k)}} .
$$

(c) The system (4.41) for determining the coefficients $\hat{d}_{1}, \ldots, \hat{d}_{k}$ reduces to

$$
\sum_{j=1}^{k} \boldsymbol{\theta}_{j} \hat{d}_{j}=-c \hat{\boldsymbol{\theta}}
$$

and can be explicitly solved as

$$
\hat{d}_{j}=\frac{d_{j}}{d_{k}}\left(\hat{d}_{k}+c \prod_{\substack{i=1 \\ i \neq j}}^{k-1} \frac{w_{i}-w_{k+1}}{w_{i}-w_{k}}\right), j=1, \ldots, k-1 .
$$

(d) The vector $\hat{\boldsymbol{\xi}}$ converges to

$$
\hat{\boldsymbol{\xi}}=-\boldsymbol{\xi}-\boldsymbol{C}_{0,0}^{-1} \boldsymbol{A}_{1,-1}\left(c w_{k+1} \hat{\boldsymbol{\theta}}+\sum_{j=1}^{k} \hat{d}_{j} w_{j} \boldsymbol{\theta}_{j}\right) .
$$

Lemmas 5.1 and 5.2 provide the ingredients to prove the absolute convergence of the series appearing in Theorem 5.1. 
Theorem 5.2 There exists a positive integer $N$, such that:

(i) The series $\sum_{\ell=0}^{\infty} \sum_{i=1}^{k^{\ell}} \alpha_{\ell, i}^{m} \sum_{j=0}^{k-1} d_{\ell, k i-j} \beta_{\ell, k i-j}^{n} \theta_{\ell, k i-j}^{(h)}$ and $\sum_{\ell=0}^{\infty} \sum_{i=1}^{k^{\ell+1}} c_{\ell, i} \alpha_{\ell+1, i}^{m} \beta_{\ell, i}^{n} \hat{\theta}_{\ell+1, i}^{(h)}$, the sum of which defines $p(h, m, n)$ for $m, n>0$, both converge absolutely for all $m>0$ and $n>0$ with $m+n>N$.

(ii) The series $\sum_{\ell=0}^{\infty} \sum_{i=1}^{k^{\ell}} \alpha_{\ell, i}^{m} \xi_{\ell, i}^{(h)}$, which defines $p(h, m, 0)$ for $m>0$, converges absolutely for all $m \geq N$.

(iii) The series

$$
\sum_{\ell=0}^{\infty} \sum_{i=1}^{k^{\ell}} \sum_{j=0}^{k-1} \frac{\alpha_{\ell, i}}{\beta_{\ell, k i-j}-\alpha_{\ell, i}} d_{\ell, k i-j} \beta_{\ell, k i-j}^{n} \theta_{\ell, k i-j}^{(h)}
$$

and

$$
\sum_{\ell=0}^{\infty} \sum_{i=1}^{k^{\ell+1}} \frac{\alpha_{\ell+1, i}}{\beta_{\ell, i}-\alpha_{\ell+1, i}} c_{\ell, i} \beta_{\ell, i}^{n} \hat{\theta}_{\ell+1, i}^{(h)}
$$

the sum of which defines $p(h, 0, n)$ for $n>0$, both converge absolutely for all $n>N$.

(iv) The series

$$
\begin{aligned}
& \sum_{m+n>N} \sum_{\ell=0}^{\infty}\left(\sum_{i=1}^{k^{\ell}} \alpha_{\ell, i}^{m} \sum_{j=0}^{k-1} d_{\ell, k i-j} \beta_{\ell, k i-j}^{n} \theta_{\ell, k i-j}^{(h)}+\sum_{i=1}^{k^{\ell+1}} c_{\ell, i} \alpha_{\ell+1, i}^{m} \beta_{\ell, i}^{n} \hat{\theta}_{\ell+1, i}^{(h)}\right) \\
& \quad+\sum_{m>N} \sum_{\ell=0}^{\infty} \sum_{i=1}^{k^{\ell}} \alpha_{\ell, i}^{m} \xi_{\ell, i}^{(h)} \\
& \quad+\sum_{n>N} \sum_{\ell=0}^{\infty}\left(\sum_{i=1}^{k^{\ell}} \sum_{j=0}^{k-1} \frac{\alpha_{\ell, i}}{\beta_{\ell, k i-j}-\alpha_{\ell, i}} d_{\ell, k i-j} \beta_{\ell, k i-j}^{n} \theta_{\ell, k i-j}^{(h)}+\sum_{i=1}^{k^{\ell+1}} \frac{\alpha_{\ell+1, i}}{\beta_{\ell, i}-\alpha_{\ell+1, i}} c_{\ell, i} \beta_{\ell, i}^{n} \hat{\theta}_{\ell+1, i}^{(h)}\right),
\end{aligned}
$$

which defines $\sum_{m+n>N} p(h, m, n)$, converges absolutely.

Proof. For $m \geq 1$ and $n \geq 1$ the series that define $p(h, m, n)$ converge absolutely if and only if

$$
\sum_{\ell=0}^{\infty} \sum_{i=1}^{k^{\ell}}\left|\alpha_{\ell, i}\right|^{m} \sum_{j=0}^{k-1}\left|d_{\ell, k i-j}\right|\left|\beta_{\ell, k i-j}\right|^{n}\left|\theta_{\ell, k i-j}^{(h)}\right|<\infty
$$

and

$$
\sum_{\ell=0}^{\infty} \sum_{i=1}^{k^{\ell+1}}\left|c_{\ell, i}\right|\left|\alpha_{\ell+1, i}\right|^{m}\left|\beta_{\ell, i}\right|^{n}\left|\hat{\theta}_{\ell+1, i}^{(h)}\right|<\infty .
$$

Consider now a fixed $m \geq 1$ and $n \geq 1$. Then, define the ratios of two consecutive terms

$$
R_{r, v}^{(\ell)}(m, n)=\frac{\left|\alpha_{\ell+1, j}\right|^{m}\left|d_{\ell+1, k(j-1)+v}\right|\left|\beta_{\ell+1, k(j-1)+v}\right|^{n}\left|\theta_{\ell+1, k(j-1)+v}^{(h)}\right|}{\left|\alpha_{\ell, i}\right|^{m}\left|d_{\ell, k(i-1)+r}\right|\left|\beta_{\ell, k(i-1)+r}\right|^{n}\left|\theta_{\ell, k(i-1)+r}^{(h)}\right|}
$$

and

$$
\hat{R}_{r, v}^{(\ell)}(m, n)=\frac{\left|c_{\ell+1, k(j-1)+v}\right|\left|\alpha_{\ell+2, k(j-1)+v}\right|^{m}\left|\beta_{\ell+1, k(j-1)+v}\right|^{n}\left|\hat{\theta}_{\ell+1, k(j-1)+v}^{(h)}\right|}{\left|c_{\ell, j}\right|\left|\alpha_{\ell+1, j}\right|^{m}\left|\beta_{\ell, j}\right|^{n}\left|\hat{\theta}_{\ell, j}^{(h)}\right|}
$$




\begin{tabular}{c|c|c}
\hline$\rho$ & $k$ & $N(\max \{\varphi(N+1), \hat{\varphi}(N+1)\})$ \\
\hline 0.15 & 2 & $0(0.030220)$ \\
& 4 & $0(0.067301)$ \\
& 9 & $0(0.143173)$ \\
\hline 0.5 & 2 & $0(0.143064)$ \\
& 4 & $0(0.167491)$ \\
& 9 & $0(0.228031)$ \\
\hline 0.85 & 2 & $0(0.191315)$ \\
& 4 & $0(0.217884)$ \\
& 9 & $0(0.271387)$ \\
\hline
\end{tabular}

Table 1: The index $N$

with $j=k(i-1)+r, i=1,2, \ldots$. As $\ell \rightarrow \infty$, the corresponding $\alpha$ 's and $\beta$ 's tend to zero and we obtain by Lemmas 5.1 and 5.2 that the ratios of two consecutive terms converges to

$$
R_{r, v}^{(\ell)}(m, n) \rightarrow R_{r, v}(m, n)=\frac{\left|w_{r}\right|^{m}\left|w_{v}\right|^{n}}{w_{k+1}^{m+n}} \frac{\left|\hat{d}_{v}\right|}{\left|d_{r}\right|}\left(\frac{\left|w_{v}\right|}{\left|w_{r}\right|}\right)^{1-h / k}
$$

and

$$
\hat{R}_{r, v}^{(\ell)}(m, n) \rightarrow \hat{R}_{r, v}(m, n)=\left(\frac{\left|w_{v}\right|}{w_{k+1}}\right)^{m+n} \frac{\left|w_{v}\right|}{\left|w_{r}\right|} \frac{\left|1-w_{r}\right|}{\left|1-w_{v}\right|} \frac{\left|\hat{d}_{v}\right|}{\left|d_{r}\right|}
$$

where the coefficients $d_{r}, \hat{d}_{v}, r, v=1, \ldots, k$ are defined in Lemma 5.2. Hence, the absolute convergence of the series is determined by the spectral radius of the corresponding matrices of rates $\boldsymbol{R}(m, n)=$ $\left(R_{r, v}(m, n)\right)_{r, v=1 \ldots, k}$ and $\hat{\boldsymbol{R}}(m, n)=\left(\hat{R}_{r, v}(m, n)\right)_{r, v=1 \ldots, k}$ (see, e.g. [4]). The spectral radius of the matrices $\boldsymbol{R}(m, n)$ and $\hat{\boldsymbol{R}}(m, n)$ only depends on $m$ and $n$ through their sum, $m+n$, so we can define $\varphi(m+n)$ and $\hat{\varphi}(m+n)$ to be the spectral radius of the matrices $\boldsymbol{R}(m, n)$ and $\hat{\boldsymbol{R}}(m, n)$, respectively. Observing that

$$
\frac{\left|w_{i}\right|}{w_{k+1}}<\frac{1}{(1+1 / k \rho)^{k}}<1, i=1, \ldots, k,
$$

we can define $N$ to be the minimal integer for which both spectral radii are less than one.

Corollary 5.2 The index $N$ is the minimal non-negative integer for which the spectral radii of the matrices $\boldsymbol{R}(N+1)$ and $\hat{\boldsymbol{R}}(N+1)$ defined by (5.8) and (5.9) are both less than one. For this minimal index $N$ the series defining $p(h, m, n)$ and $\sum_{m, n} p(h, m, n)$ for $m \geq 0$ and $n \geq 0$ with $m+n>N$, presented in Theorem 5.1, converge absolutely.

Remark 5.1 In general, the index $N$ is small. In the special case of Poisson arrivals join the shorter queue $(k=1)$ it follows that $N=0$. The same seams to apply for $k \geq 2$ according to our numerical findings.

We are now in a position to state the main result of the paper. 
Theorem 5.3 For all states $(h, m, n), h=0,1, \ldots, k-1, m, n \geq 0$ and $m+n>N$ including $m=N$ and $n=0$,

$$
\begin{aligned}
\boldsymbol{p}(m, n)= & C^{-1} \sum_{\ell=0}^{\infty} \sum_{i=1}^{k^{\ell}} \alpha_{\ell, i}^{m} \sum_{j=0}^{k-1} d_{\ell, k i-j} \beta_{\ell, k i-j}^{n} \boldsymbol{\theta}_{\ell, k i-j} \\
& +C^{-1} \sum_{\ell=0}^{\infty} \sum_{i=1}^{k^{\ell+1}} c_{\ell, i} \alpha_{\ell+1, i}^{m} \beta_{\ell, i}^{n} \hat{\boldsymbol{\theta}}_{\ell+1, i}, m>0, n>0 \\
\boldsymbol{p}(m, 0)= & C^{-1} \sum_{\ell=0}^{\infty} \sum_{i=1}^{k^{\ell}} \alpha_{\ell, i}^{m} \boldsymbol{\xi}_{\ell, i}, m>0, \\
\boldsymbol{p}(0, n)= & C^{-1} \sum_{\ell=0}^{\infty} \sum_{i=1}^{k^{\ell}} \sum_{j=0}^{k-1} \frac{\alpha_{\ell, i}}{\beta_{\ell, k i-j}-\alpha_{\ell, i}} d_{\ell, k i-j} \beta_{\ell, k i-j}^{n} \boldsymbol{\theta}_{\ell, k i-j} \\
& +C^{-1} \sum_{\ell=0}^{\infty} \sum_{i=1}^{k^{\ell+1}} \frac{\alpha_{\ell+1, i}}{\beta_{\ell, i}-\alpha_{\ell+1, i}} c_{\ell, i} \beta_{\ell, i}^{n} \hat{\boldsymbol{\theta}}_{\ell+1, i}, n>0 \\
\boldsymbol{p}(0,0)= & C^{-1} \frac{1}{2 k \rho} \boldsymbol{T}_{k} \boldsymbol{p}(0,1),
\end{aligned}
$$

where $C$ is the normalizing constant and $\boldsymbol{T}_{k}$ denotes a $k \times k$ lower triangular matrix, with elements $(i, j), i \geq j, j=1, \ldots, k$, equal to one and zeros elsewhere.

Proof. Define $\mathcal{L}^{(N)}$ to be the set of states $(m, n)$ with $m+n>N$ including state $(m, n)=(N, 0)$. Then $\mathcal{L}^{(N)}$ is the set on which the series of Theorem 5.1 converge absolutely. The restricted stochastic process on the set $\mathcal{L}^{(N)}$ with transition rates as given in Fig. 5 is an irreducible Markov process, whose associated equilibrium equations are identical to the equations of the original unrestricted process on the set $\mathcal{L}^{(N)}$, except for the equation of state $(N, 0)$. Hence, the process restricted to $\mathcal{L}^{(N)}$ is ergodic so that the series of Theorem 5.1 can be normalized to produce the equilibrium distribution of the restricted process on $\mathcal{L}^{(N)}$. Since the set $\mathscr{H} \times \mathbb{N}_{0} \times \mathbb{N}_{0} \backslash \mathcal{L}^{(N)}$ is finite, it follows that the original process is ergodic and relating appropriately the equilibrium probabilities of the unrestricted and restricted process proves Theorem 5.3.

\section{Conclusions}

We have studied the symmetric shorter queue problem with Erlang arrivals and have proved that the equilibrium distribution of the queue lengths can be represented by an infinite series of product-forms by using a generalization of the compensation approach. These analytical results offer numerically efficient algorithms, mainly due to the exponential convergence of the series.

This paper has demonstrated that the compensation approach can work for three-dimensional Markov chains, whose state space consists of a $k$-layered quarter plane. We believe that the same approach can be used to obtain the equilibrium distribution in the case of phase-type arrivals, and even for the more general Markovian arrival process (MAP). 


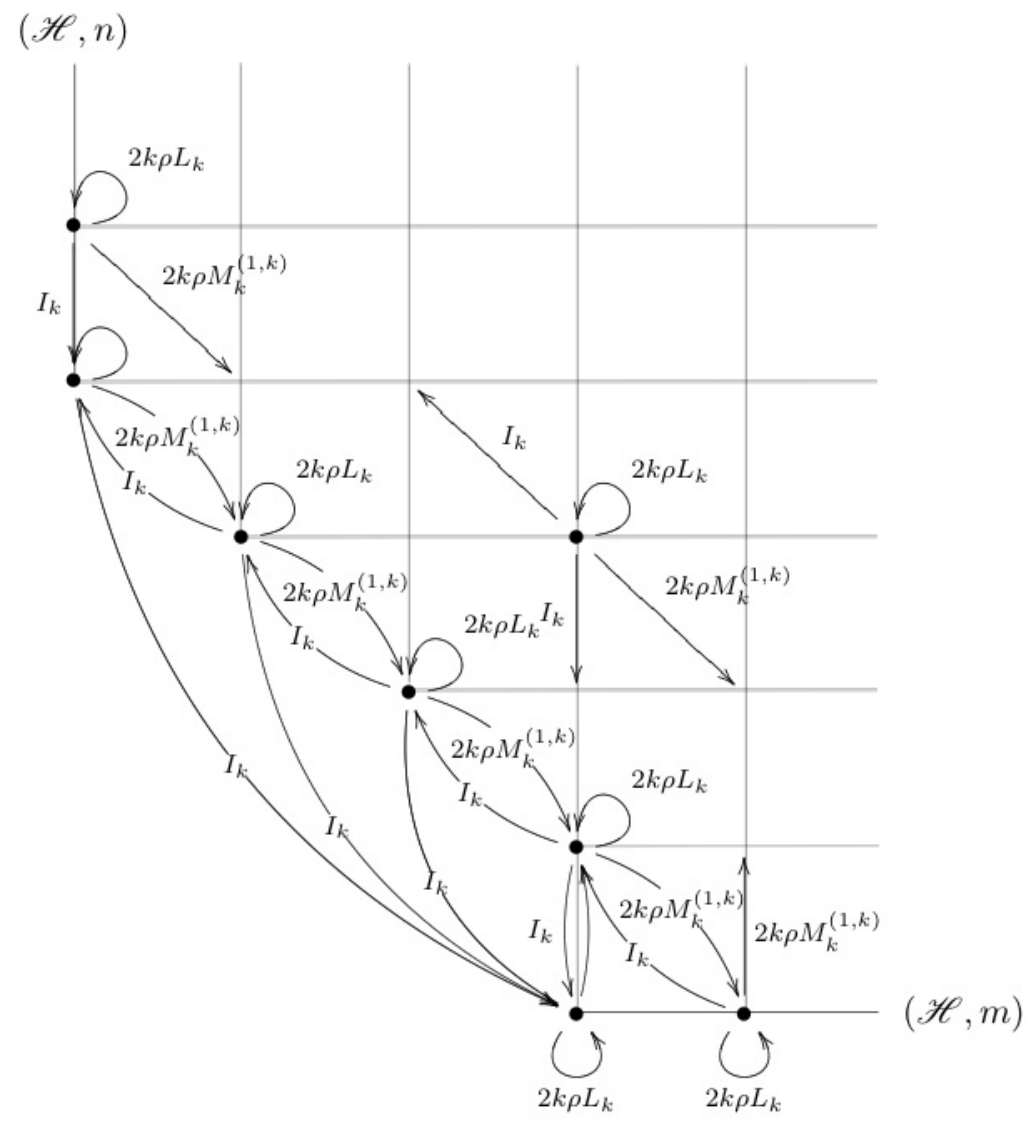

Figure 5: $\mathcal{L}^{(4)}$ transition rate diagram with $\mathscr{H}=\{0,1, \ldots, k-1\}$.

\section{References}

[1] I.J.B.F. Adan. A Compensation Approach for Queueing Problems. PhD dissertation, Technische Universiteit Eindhoven, Eindhoven, 1991.

[2] I.J.B.F. Adan, O.J. Boxma, and J. Resing. Queueing models with multiple waiting lines. Queueing Syst., 37(1-3):65-98, 2001.

[3] I.J.B.F. Adan, J. Wessels, and W.H.M. Zijm. Analysis of the symmetric shortest queue problem. Comm. Statist. Stochastic Models, 6(4):691-713, 1990.

[4] I.J.B.F. Adan, J. Wessels, and W.H.M. Zijm. Analysis of the asymmetric shortest queue problem. Queueing Syst., 9(1):1-58, 1991.

[5] I.J.B.F. Adan, J. Wessels, and W.H.M. Zijm. A compensation approach for two-dimensional Markov processes. Adv. in Appl. Probab., 25(4):783-817, 1993.

[6] A. Bostan and M. Kauers. The complete generating function for Gessel's walk is algebraic. Proc. Amer. Math. Soc., 432:3063-3078, 2010. 
[7] M. Bousquet-Mélou and M. Mishna. Walks with small steps in the quarter plane. In Algorithmic Probability and Combinatorics, M. Lladser, R. Maier, M. Mishna, A. Rechnitzer, (Eds.), 1-41. Amer. Math. Soc., Providence, RI, 2010.

[8] J.W. Cohen and O.J. Boxma. Boundary Value Problems in Queueing System Analysis, NorthHolland, Amsterdam, 1983.

[9] G. Fayolle, R. Iasnogorodski, and V. Malyshev. Random Walks in the Quarter Plane. SpringerVerlag, Berlin, 1999.

[10] G. Fayolle and K. Raschel. On the holonomy or algebraicity of generating functions counting lattice walks in the quarter-plane. Markov Process. Related Fields, 16(3):485-496, 2010.

[11] P. Flajolet and R. Sedgewick. Analytic Combinatorics. Cambridge University Press, Cambridge, 2009.

[12] S.G. Foss and N.I. Chernova On optimality of FCFS discipline in multi-channel queueing systems and networks. Siberian Math. J., 42:372-385, 2001.

[13] F.G. Foster. On stochastic matrices associated with certain queueing processes. Ann. Math. Stat., 24:355-360, 1953.

[14] F.A. Haight. Two queues in parallel. Biometrika, 45(3-4):401-410, 1958.

[15] J.F.C. Kingman. Two similar queues in parallel. The Annals of Mathematical Statistics, 32(4):1314-1323, 1961.

[16] I. Kurkova and K. Raschel. Explicit expressions for the generating function counting Gessel's walk. Adv. in Appl. Math., to appear.

[17] Z. Liu, P. Nain and D. Towsley. Sample path methods in the control of queues. Queueing Systems, 21:293-335, 1995.

[18] V. Malyshev. Positive random walks and Galois theory. Uspehi Mat. Nauk, 26(1(157)):227-228, 1971.

[19] M. Marden. Kakeya's Problem on the Zeros of the Derivative of a Polynomial. Transactions of the American Mathematical Society, 45(3):355-368, 1939.

[20] M. Mishna and A. Rechnitzer. Two non-holonomic lattice walks in the quarter plane. Theor. Comput. Sci., 410(38-40):3616-3630, 2009.

[21] M. Miyazawa. Light tail asymptotics in multidimensional reflecting processes for queueing networks. TOP. DOI: 10.1007/s11750-011-0179-7, 2011.

[22] K. Raschel. Counting walks in a quadrant: a unified approach via boundary value problems. Arxiv: 1003.1362:1-23, 2010.

[23] J.A.C. Resing and R. Rietman. The $M / M / 1$ queue with gated random order of service. Statist. Neerlandica, 58(1):97-110, 2004. 
[24] Y. Sakuma, M. Miyazawa and Y.Q. Zhao. Decay rate for the $P H / M / 2$ queue with shortest queue discipline. Queueing Systems, 53:189-201, 2006.

[25] T. Sheil-Small. Complex Polynomials. Cambridge University Press, 2002.

[26] E.B. Vinberg. A Course in Algebra. American Mathematical Society, Providence, RI, 2003.

\section{A Determining the starting $\alpha$ for the modified model}

Lemma A.1 For $\rho<1$, the product-form

$$
\hat{p}(h, m, n)=\sigma^{2 m} \hat{q}_{h}(n), h=0,1, \ldots, k-1, m \geq-n, n \geq 0,
$$

with $\sigma$ the unique root of (3.4) inside the open unit circle and $\left\{\hat{q}_{h}(n)\right\}_{n \in \mathbb{N}_{0}}$ such that $\sum_{n=0}^{\infty} \sigma^{-2 n} \hat{q}_{h}(n)<$ $\infty$, is the unique (up to a constant) solution of the equilibrium equations (2.10)-(2.12).

Proof. We consider levels of the form

$$
(L, h)=\{(h, m, n): 2 m+n=L \geq 0\}, h=0,1, \ldots k .
$$

$$
\begin{gathered}
h=0 \\
h=1 \\
h=k-2 \\
h=k-1
\end{gathered}
$$

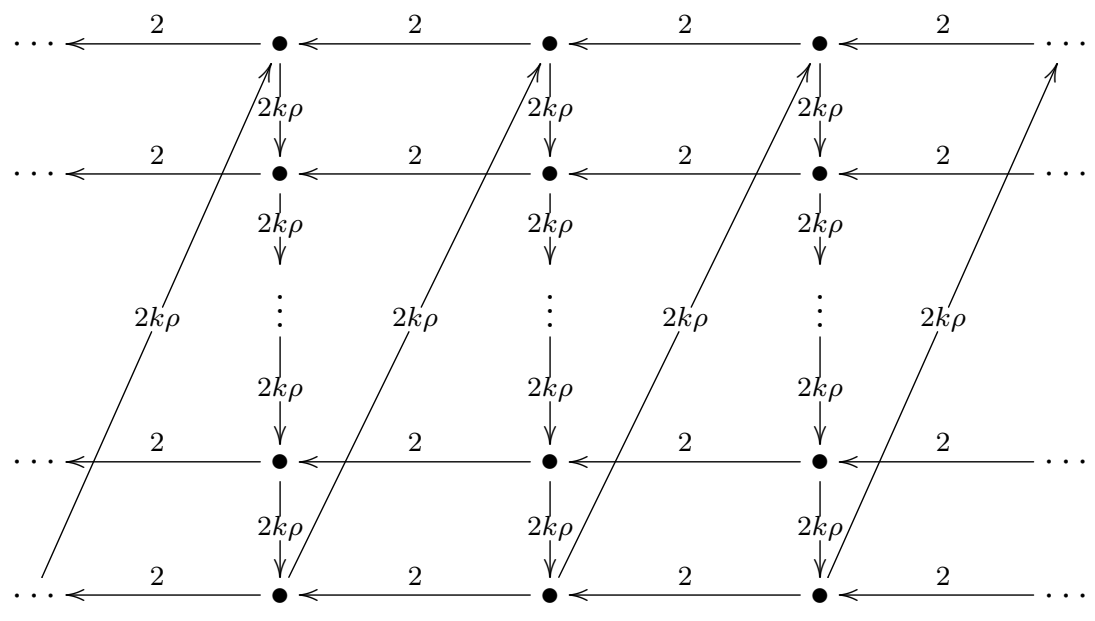

$$
L=\ell-1 \quad L=\ell \quad L=\ell+1
$$

Figure 6: Transition rate diagram of the levels $\{(L, h), L \in \mathbb{N}, h=0,1, \ldots, k-1\}$ of the modified model.

The equilibrium equations between the levels are given by (see Fig. 6)

$$
\begin{aligned}
& (k \rho+1) \sum_{2 m+n=L} \hat{p}(0, m, n)=k \rho \sum_{2 m+n=L-1} \hat{p}(k-1, m, n)+\sum_{2 m+n=L+1} \hat{p}(0, m, n), \\
& (k \rho+1) \sum_{2 m+n=L} \hat{p}(h, m, n)=k \rho \sum_{2 m+n=L} \hat{p}(h-1, m, n)+\sum_{2 m+n=L+1} \hat{p}(h, m, n),
\end{aligned}
$$


for $h=1, \ldots, k-1$. Substituting (4.1) into (A.2) and (A.3) yields

$$
\begin{aligned}
& (k \rho+1) \sum_{2 m+n=L} \alpha^{m} \hat{q}_{0}(n)=k \rho \sum_{2 m+n=L-1} \alpha^{m} \hat{q}_{k-1}(n)+\sum_{2 m+n=L+1} \alpha^{m} \hat{q}_{0}(n), \\
& (k \rho+1) \sum_{2 m+n=L} \alpha^{m} \hat{q}_{h}(n)=k \rho \sum_{2 m+n=L} \alpha^{m} \hat{q}_{h-1}(n)+\sum_{2 m+n=L+1} \alpha^{m} \hat{q}_{h}(n),
\end{aligned}
$$

for $h=1, \ldots, k-1$. Iterating equation (A.5) gives

$$
\sum_{2 m+n=L} \alpha^{m} \hat{q}_{0}(n)=\sum_{i=0}^{h}\left(\begin{array}{c}
h \\
i
\end{array}\right)(-1)^{i} \frac{(k \rho+1)^{h-i}}{(k \rho)^{h}} \sum_{2 m+n=L+i} \alpha^{m} \hat{q}_{h}(n),
$$

for $h=1, \ldots, k-1$. Substituting equation (A.6) for $h=k-1$ into (A.4) yields

$$
\begin{aligned}
\sum_{i=0}^{k-1}\left(\begin{array}{c}
k-1 \\
i
\end{array}\right)(-1)^{i} \frac{(k \rho+1)^{k-i}}{(k \rho)^{k-1}} & \sum_{2 m+n=L+i} \alpha^{m} \hat{q}_{k-1}(n)=k \rho \sum_{2 m+n=L-1} \alpha^{m} \hat{q}_{k-1}(n) \\
& -\sum_{i=1}^{k}\left(\begin{array}{c}
k-1 \\
i-1
\end{array}\right)(-1)^{i} \frac{(k \rho+1)^{k-i}}{(k \rho)^{k-1}} \sum_{2 m+n=L+i} \alpha^{m} \hat{q}_{k-1}(n) .
\end{aligned}
$$

Observing that

$$
\sum_{2 m+n=L} \alpha^{m} \hat{q}_{k-1}(n)=\sum_{2 m+n=L} \sigma^{2 m} \hat{q}_{k-1}(n)=\sum_{\substack{j+n=L \\ j \text { odd }}} \sigma^{j} \hat{q}_{k-1}(n)=\sum_{\substack{n=0 \\ L-n \text { odd }}}^{L} \sigma^{L-n} \hat{q}_{k-1}(n)
$$

with $\sigma=\alpha^{2}$, and equating all coefficients of $\hat{q}_{k-1}(n)$ in (A.7) leads, for $0 \leq n \leq L-1$,

$$
\begin{aligned}
(k \rho)^{k} & =\sum_{i=0}^{k-1}\left(\begin{array}{c}
k-1 \\
i
\end{array}\right)(-1)^{i}(k \rho+1)^{k-i} \sigma^{i+1}+\sum_{i=1}^{k}\left(\begin{array}{c}
k-1 \\
i-1
\end{array}\right)(-1)^{i}(k \rho+1)^{k-i} \sigma^{i+1} \\
& =\sum_{i=0}^{k}\left(\begin{array}{c}
k \\
i
\end{array}\right)(-1)^{i}(k \rho+1)^{k-i} \sigma^{i+1}=\sigma(k \rho+1-\sigma)^{k},
\end{aligned}
$$

which is exactly equation (3.4).

\section{B Appendix}

Lemma B.1 For all $x$, with $|x|<1$,

$$
|1+\sqrt{1 \pm x}| \geq 1+\sqrt{1-|x|}>1>|x| .
$$

Proof. Taylor expansion of the square root yields

$$
1-\sqrt{1-x}=\sum_{n=1}^{\infty} \frac{(2 n) !}{(2 n-1)(n !)^{2} 4^{n}} x^{n},|x|<1 .
$$

Then

$$
\begin{aligned}
|1+\sqrt{1-x}| & =\left|2-\sum_{n=1}^{\infty} \frac{(2 n) !}{(2 n-1)(n !)^{2} 4^{n}} x^{n}\right| \\
& \geq 2-\sum_{n=1}^{\infty} \frac{(2 n) !}{(2 n-1)(n !)^{2} 4^{n}}|x|^{n} \\
& \stackrel{(B .2)}{=} 1+\sqrt{1-|x|}>1 .
\end{aligned}
$$




\section{Appendix}

In what follows we will assume that the $\alpha$ 's are in absolute value smaller than or equal to the starting solution $\sigma^{2}$, with $\sigma$ the unique root in $(0,1)$ of $(3.4)$. However, for $0<\rho<1$ one can easily verify that $\sigma<\rho$, so we will assume that $|\alpha|<\rho^{2}$.

Lemma C.1 For $\rho<1$ and for every $\alpha$, with $|\alpha| \in\left(0, \rho^{2}\right)$, the equation $\operatorname{det}(\boldsymbol{D}(\alpha, \beta))=0$ assumes the form

$$
\left(2(k \rho+1) \beta-\beta^{2}-\alpha\right)^{k} \alpha-(2 k \rho)^{k} \beta^{k+1}=0,
$$

and has exactly $k$ simple roots in the $\beta$-plane with $0<|\beta|<|\alpha| / 2$.

Proof. Equation (4.20) is a polynomial in $\beta$ of degree $2 k$ and we will show that exactly $k$ roots are inside the open circle of radius $|\alpha| / 2$. We divide both sides of equation (4.20) by $\alpha^{k+1}$ and set $z=\beta / \alpha$ which gives

$$
f_{1}^{k}(z)-(2 k \rho)^{k} z^{k+1}=0
$$

with $f_{1}(z)=2(k \rho+1) z-\alpha z^{2}-1$. Then $f_{1}(z)$ has two roots:

$$
z_{ \pm}=\frac{k \rho+1 \pm \sqrt{(k \rho+1)^{2}-\alpha}}{\alpha} .
$$

For $0<\rho<1$, we observe that $z_{+}$is located outside the open unit circle, while

$$
\begin{aligned}
\left|z_{-}\right| & =\frac{k \rho+1}{|\alpha|}\left|1-\sqrt{1-\frac{\alpha}{(k \rho+1)^{2}}}\right|=\frac{1}{k \rho+1} \frac{1}{\left|1+\sqrt{1-\frac{\alpha}{(k \rho+1)^{2}}}\right|} \\
& \stackrel{(B .1)}{<} \frac{1}{k \rho+1} \frac{1}{1+\sqrt{1-\frac{|\alpha|}{(k \rho+1)^{2}}}}<\frac{1}{2} .
\end{aligned}
$$

Furthermore, for $|z|=1 / 2$,

$$
\begin{aligned}
\left|f_{1}^{k}(z)\right| & =\left|2(k \rho+1) z-\alpha z^{2}-1\right|^{k}>\left.|2(k \rho+1)| z|-| \alpha|| z\right|^{2}-\left.1\right|^{k} \\
& =\left(2 k \rho-\frac{|\alpha|}{2}\right)^{k}\left(\frac{1}{2}\right)^{k}>\left(2 k \rho-\frac{\rho^{2}}{2}\right)^{k}\left(\frac{1}{2}\right)^{k} \\
& =\left(1-\frac{\rho}{4 k}\right)^{k}(k \rho)^{k}>\left(1-\frac{1}{8}\right)^{2}(k \rho)^{k} \\
& >(2 k \rho)^{k}\left(\frac{1}{2}\right)^{k+1}=\left|g_{1}(z)-f_{1}^{k}(z)\right| .
\end{aligned}
$$

Hence by Rouché's theorem $f_{1}^{k}(z)-(2 k \rho)^{k} z^{k+1}$ has exactly $k$ roots inside $|z|=1 / 2$, which completes the proof.

According to Lemma C.1 all $\beta$ roots are located in the open circle with radius $\rho^{2} / 2$, so we assume that $|\beta|<\rho^{2} / 2$. 
Lemma C.2 For $\rho<1$ and for every $\beta$, with $|\beta| \in\left(0, \rho^{2} / 2\right)$, the equation $\operatorname{det}(\boldsymbol{D}(\alpha, \beta))=0$ assumes the form

$$
\left(2(k \rho+1) \beta-\beta^{2}-\alpha\right)^{k} \alpha-(2 k \rho)^{k} \beta^{k+1}=0,
$$

and has exactly one root in the $\alpha$-plane with $0<|\alpha|<3|\beta| / 4$.

Proof. Equation (4.20) is a polynomial in $\alpha$ of degree $k+1$ and we will show that there is only one root inside the open circle of radius $3|\alpha| / 4$. We divide both sides of equation (4.20) by $\beta^{k+1}$ and set $x=\alpha / \beta$, which gives

$$
f_{2}(x)-(2 k \rho)^{k}=0
$$

with $f_{2}(x)=x(2 k \rho+2-\beta-x)^{k}$. Then $f_{2}(x)$ has the zero root of multiplicity one and the root $2 k \rho+2-\beta$ of multiplicity $k$ larger than the radius $3 / 4$. Furthermore, for $|x|=3 / 4$ we have that

$$
\begin{aligned}
\left|f_{2}(x)\right| & =\left|x(2 k \rho+2-\beta-x)^{k}\right| \geq \frac{3}{4}\left(2 k \rho+2-|\beta|-\frac{3}{4}\right)^{k} \\
& =\frac{3}{4}\left(2 k \rho+\frac{5}{4}-|\beta|\right)^{k}=\frac{3}{4}(2 k \rho)^{k}\left(1+\frac{5}{8 k \rho}-\frac{|\beta|}{2 k \rho}\right)^{k} \\
& >\frac{3}{4}(2 k \rho)^{k}\left(1+\frac{5}{8 k \rho}-\frac{\rho}{4 k}\right)^{k}>(2 k \rho)^{k} \frac{3}{4}\left(1+\frac{5}{16}-\frac{1}{8}\right)^{2}>(2 k \rho)^{k} .
\end{aligned}
$$

Hence, by Rouché's theorem $f_{2}(x)-(2 k \rho)^{k}$ has exactly one root in $|x|<3 / 4$, which completes the proof. 